\title{
A Comparative Evaluation of Numerical and Analytical Solutions to the Biadhesive Single-Lap Joint
}

\author{
Halil Özer' and Özkan Öz ${ }^{2}$ \\ ${ }^{1}$ Mechanical Engineering Department, Faculty of Mechanical Engineering, Yıldız Technical University, Yıldız, \\ Beşiktaş, 34349 Istanbul, Turkey \\ ${ }^{2}$ Machine Education Division, Faculty of Technical Education, Karabuk University, 78050 Karabuk, Turkey \\ Correspondence should be addressed to Halil Özer; hozer@yildiz.edu.tr
}

Received 23 December 2013; Accepted 15 January 2014; Published 10 March 2014

Academic Editor: Evangelos J. Sapountzakis

Copyright (C) 2014 H. Özer and Ö. Öz. This is an open access article distributed under the Creative Commons Attribution License, which permits unrestricted use, distribution, and reproduction in any medium, provided the original work is properly cited.

\begin{abstract}
This paper attempts to address the detailed verification of Zhao's analytical solution including the moment effect with the two- and three-dimensional finite element results. Zhao compared the analytical results with only the 2D FEA results and used the constant bond-length ratio for the biadhesive bondline. In this study, overlap surfaces of the adherends and the adhesives were modelled using surface-to-surface contact elements. Both analytical and numerical analyses were performed using four different biadhesive bondline configurations. The 3D FEA results reveal the existence of complex stress state at the overlap ends. However, the general results show that analytical and numerical results were in a good agreement.
\end{abstract}

\section{Introduction}

Structural adhesives have been used extensively in the space, aviation, automotive, and naval industries. Single-lap joints are the most widely used adhesive joints and have been investigated by many researchers [1-8]. Techniques reducing peel and shear stress concentrations are tapering the adherend, forming an adhesive fillet, changing the lap joint geometry, and so forth. However, these techniques can have some disadvantages. For example, tapering the adherend damage fiber structure of the fiber-reinforced composites and forming an adhesive fillet are quite difficult when low viscosity adhesives are used.

An alternative technique is to use a combination of stiff and flexible adhesives along the overlap region. The stiff adhesive should be located in the middle and flexible adhesive at the ends. Different names for this type of joint are used in the literature such as mixed-adhesive, biadhesive, and hybrid-adhesive joints. The joints bonded with biadhesive transfer the stresses from the ends towards the centre of the overlap more than the joints bonded with a monoadhesive alone. Therefore, high stress concentrations at the ends can be reduced by using this technique.
Biadhesive joints have been studied in a limited number of papers in the literature. Raphael's early paper [9] related to the biadhesive joint showed the possible benefits of using a mixed-modulus bondline. He neglected the peel stress effect in his model. Srinivas [10] investigated the application of combined flexible and stiff adhesives in the bondline. His model has an ability to include dissimilar as well as composite adherends. Pires et al. [11] investigated a biadhesive joint with aluminium adherends using both experimental and numerical (finite element) techniques. They proved that joint strength can be optimized by choosing appropriate joint geometry and material. Fitton and Broughton [12] used a linear elastic 2D finite element method (FEM) to compare hybrid and monoadhesive bondlines. They showed that significant strength improvement can be obtained if joint failure stresses are considerably less than the shear strength of the adhesive. Three-dimensional finite element analyses of hybrid-adhesive joints under cleavage and tensile load were carried out by Kong et al. [13]. They showed that maximum stresses along the bondline can be decreased by using appropriate bond-length ratios. Also, they emphasized that it is necessary to take into account the change of loading modes when optimizing the stress distributions of 
the biadhesive joint. Kumar [14] investigated the effects of functionally graded bondlines on the stress components in tubular joints. He suggested controlling the modulus of the adhesive spatially for optimization of the peel and shear strengths. Variable flexibility and strength along the overlap length were described as an ideal adhesive joint by da Silva and Lopes [15]. They concluded that, if the ductile adhesive has a joint strength lower than that of the brittle adhesive, a mixed-adhesive joint with both adhesives gives a joint strength higher than the joint strength of the adhesives used individually. This synergetic effect can be explained by the shear stress distribution of the adhesive at failure. The effect of the biadhesive bondline on the stress distribution of weldbonded joints was studied by You et al. [16]. They showed that the load bearing capacity of the weld-bonded joints may be increased by transferring some parts of stress from the adhesive layer to the weld nugget. Da Silva and Adams [17] studied titanium/titanium and titanium/composite doublelap joints formed using a hybrid-adhesive bondline. They showed that the suitable combination of two adhesives gives a better performance over a wide temperature range $\left(-55^{\circ} \mathrm{C}\right.$ to $200^{\circ} \mathrm{C}$ ) than a high temperature adhesive alone for a joint with dissimilar adherends. Temiz [18] studied the effect of a hybrid-adhesive bondline on the strength of double strap joints subjected to external bending moments. He concluded that stress concentration at the overlap ends decreases by applying the flexible adhesive towards the ends of the overlap in bonded joints. By using the flexible adhesive in biadhesive joints, the strains do not increase significantly when compared with increase in predicted failure load. This indicates that the stiffer adjacent adhesive has the constraining effect on the strain in the flexible adhesive. Das Neves et al. [19] developed an analytical model for hybrid-adhesive single and double lap joints subjected to low and high temperatures. They compared the solutions of the analytical model with a finite element analysis and observed only small differences close to the overlap ends where the maximum adhesive shear and peel stresses occurred. Pires et al. [20] discussed the failure mechanism of biadhesive joints. Their results showed an increase in shear strength of the biadhesive-bonded joints compared with those in which monoadhesives were used over the full length of the bondline. The increase in the apparent lap-shear strength was predicted through finite element model. Kumar and Pandey [21] performed the twodimensional and three-dimensional nonlinear (geometric and material) finite element analyses of adhesively bonded single-lap joints having modulus-graded bondline under monotonic loading conditions. The adhesives were modelled as an elastoplastic multilinear material, while the substrates were regarded as both linear elastic and bilinear elastoplastic material. They observed that the static strength was higher for joints with biadhesive bondlines compared to those with monoadhesive bondlines. Effects of load level and bondline thickness on stress distribution in the biadhesive bondline were also studied.

Carbas et al. [22] developed a functionally modified adhesive in order to have mechanical properties that vary gradually along the overlap, allowing a more uniform stress distribution along the overlap and to reduce the stress concentrations at the ends of the overlap. Grading was achieved by induction heating, giving a graded cure of the adhesive. Analytical analyses were performed to predict the failure load of the joints with graded cure and isothermal cure. The functionally graded joint was found to have a higher joint strength compared to the cases where the adhesive was cured uniformly at low temperature or at high temperature. The simple analytical analysis proposed by the authors was shown to be a valid tool to predict the maximum failure load of the functionally graded joint. Carbas et al. [23] studied a functionally modified adhesive. Simple analytical model to study the performance of the functionally graded joints was developed. The differential equation of this model was solved by a power series. Finite element analysis was performed to validate the analytical model developed. The joints with the adhesive properties functionally modified along the overlap showed a high strength when compared with the joints with homogeneous adhesive properties along the overlap. Both shear stress distributions of the bond line were found to have a similar behavior comparing the analytical analysis by Power series expansion with the numerical analysis by a FE analysis. Bavi et al. [24] optimized the geometry of the overlap in mixed adhesive single- and double-lap joints using a modified version of Bees and Genetic Algorithms (BA and GA). Four and five optimization variables were considered within bi- and triadhesive joint configurations, respectively. Eventually, the efficiencies of the two employed algorithms, namely, modified BA and GA, were compared with each other. Most optimal joint configurations were described by a long adhesive bond length and thick layers of adherends. Comparing a modified version of BA (MBA) and $\mathrm{GA}$, it was observed that the first algorithm has a significant robustness, producing a $100 \%$ success rate in all considered cases. They concluded that the proposed MBA proved to be a very suitable candidate for these types of engineering problems.

Many closed-form solutions are available in the open literature. The early analytical model was developed by Volkersen [25]. However, Volkersen method, known as the shear-lag model, neglected the rotation of the joint. A lot of improvements were made over the following seventy years including the addition of the rotation of the adherends, adhesive plasticity, and the adherend shear deformation $[26,27]$. Recent contribution has been made by Zhao. He proposed some closed-form solutions to evaluate the stress components along the adhesive bondline and then extended these solutions to the biadhesive bondline by taking into account the bending effect [28].

In this study, after reviewing the current literature on the existing analytical models for the biadhesive single-lap joints, some expressions related to stress components for the single-lap joint with biadhesive bondlines were derived by following the same steps as Zhao's solutions [28]. Deformations due to the bending effect which causes the joint to rotate were included in the formulations. A MAPLE program was written. Prepared MAPLE program employs these expressions to calculate stress components. The validity of the analytical results was assessed by comparing the $2 \mathrm{D}$ and 3D FEA results for the mono- and biadhesive bondlines. 
The analytical model was based on the plane elasticity and some restrictive assumptions, but the state of stress in the joint has the three-dimensional nature. Especially, the adhesive peel stress is sensitive to the three-dimensional effects. Therefore, the three-dimensional FEM model can provide more accurate predictions for comparing the results without introducing any simplification at the modelling procedure. In this study, the 3D FEM model is based on the surface-tosurface contact elements. Aluminum adherends were bonded with stiff and flexible adhesives. The stiff adhesive was located in the middle and the flexible adhesive was located at the ends of the overlap. The overlap length, the adhesive thickness, the adherend thickness, and modulus of stiff and flexible adhesives were kept constant. Both analytical and numerical analyses were performed using four different bond-length ratios for the biadhesive joint. The effect of biadhesive and monoadhesive bondlines on the peel stress (transverse normal stress) and shear stress distributions was investigated. The numerical analyses were performed using the Ansys finite element code. The results of both FEM analyses and analytical solutions were compared.

It must be stated that this paper aims especially to compare the analytical solutions with the 3D FEA solutions and to show the three-dimensional nature of the state of stress in the joint. Zhao [28] compared stress results predicted by the closed-form expressions only with the 2D FEA results. The bond-length ratio for the biadhesive bondline was kept constant in his study.

\section{Analytical Evaluation of Stress Components for Biadhesive Joint}

The biadhesive bondline can be modeled as three individual regions according to their shear modulus components (Figure 1). The stiff adhesive was located in the middle and flexible adhesive at the ends of the overlap. Two adherends with the thicknesses of $\delta_{1}$ and $\delta_{2}$ were bonded with an adhesive layer with a thickness of $\delta_{3}$, where $\delta_{4}=\delta_{3}$. The regions I and III at the overlap ends are the left and right flexible adhesive regions, respectively. The region II at the centre of the overlap is the stiff adhesive region. The two ends of the adherends are simply supported, and right end is subjected to an axial load $F$. (As seen in Figure 1, the $x$-axis passes through the midplane of the adhesive layer.)

In Figure $1 l_{f}$ is the flexible adhesive length and $l_{s}$ is the stiff adhesive length, where $l_{f}=(l-s)$ and $l_{s}=2 s$. The upper and the lower adherends are denoted by the subscripts 1 and 2, respectively. The flexible and stiff adhesives are denoted by the subscripts 3 and 4 , respectively. Then, $E_{i}$ and $G_{i}(i=1,2,3,4)$ are the modulus of elasticity and shear modulus of the four individual components. The total overlap length is $2 \ell$. The joint width, perpendicular to the $(x, y)$-plane, is $b . V_{0}$ and $M_{0}$ are the shear force and bending moment acting on the ends of the upper and lower adherends, respectively (Figure 1(b)). A differential section $d x$ can be cut out from the overlap region of the biadhesive joint as shown in Figure 2.

In Figure $2 \sigma_{3 y}$ is the peel stress at the upper and lower interfaces of the adhesive. $F_{i x}, V_{i}$ and $M_{i}(i=1,2)$ are the tensile forces, shear forces, and bending moments related to the upper and lower adherends, respectively.

The distributions of the longitudinal shear stresses and displacements of adherends 1 and 2 are illustrated in Figure 3. A linear shear stress and strain distributions through the thickness of the adherends is assumed. Two local coordinate systems, $y_{1}$ and $y_{2}$, were introduced, where $y_{1}$ and $y_{2}$ are the distances from the top of the upper and lower adherends, respectively. Free surface stress conditions are considered.

The detailed derivation of the stress components for the bi-adhesive joint along the bondline was given in the Appendix. For the three regions (I, II, and III) shown in Figure 1, the three sets of expressions are given as follows:

Region I $(-l \leq x \leq-s)$

$$
\begin{gathered}
\sigma_{1 x}^{\imath}=A_{1} \sinh \left(\lambda_{1} x\right)+A_{2} \cosh \left(\lambda_{1} x\right)+A_{3} x+A_{4}, \\
\tau_{3 x}^{\imath}=-\delta_{0}\left(A_{1} \lambda_{1} \cosh \left(\lambda_{1} x\right)+A_{2} \lambda_{1} \sinh \left(\lambda_{1} x\right)+A_{3}\right), \\
\sigma_{3 y}^{\imath}=B_{1} \sinh \left(\omega_{1} x\right) \sin \left(\omega_{1} x\right)+B_{2} \sinh \left(\omega_{1} x\right) \cos \left(\omega_{1} x\right) \\
+B_{3} \cosh \left(\omega_{1} x\right) \sin \left(\omega_{1} x\right)+B_{4} \cosh \left(\omega_{1} x\right) \cos \left(\omega_{1} x\right)
\end{gathered}
$$

Region II $(-s \leq x \leq s)$

$$
\begin{gathered}
\sigma_{1 x}^{u l}=A_{5} \sinh \left(\lambda_{2} x\right)+A_{6} \cosh \left(\lambda_{2} x\right)+A_{7} x+A_{8}, \\
\tau_{4 x}^{\imath l}=-\delta_{0}\left(A_{5} \lambda_{2} \cosh \left(\lambda_{2} x\right)+A_{6} \lambda_{2} \sinh \left(\lambda_{2} x\right)+A_{7}\right), \\
\sigma_{4 y}^{\imath l}=B_{5} \sinh \left(\omega_{2} x\right) \sin \left(\omega_{2} x\right)+B_{6} \sinh \left(\omega_{2} x\right) \cos \left(\omega_{2} x\right) \\
+B_{7} \cosh \left(\omega_{2} x\right) \sin \left(\omega_{2} x\right)+B_{8} \cosh \left(\omega_{2} x\right) \cos \left(\omega_{2} x\right) .
\end{gathered}
$$

Region III $(s \leq x \leq l)$

$$
\begin{aligned}
\sigma_{1 x}^{\imath n}= & A_{9} \sinh \left(\lambda_{1} x\right)+A_{10} \cosh \left(\lambda_{1} x\right)+A_{11} x+A_{12}, \\
\tau_{3 x}^{\imath u}= & -\delta_{0}\left(A_{9} \lambda_{1} \cosh \left(\lambda_{1} x\right)+A_{10} \lambda_{1} \sinh \left(\lambda_{1} x\right)+A_{11}\right) \\
\sigma_{3 y}^{\imath \imath \imath}= & B_{9} \sinh \left(\omega_{1} x\right) \sin \left(\omega_{1} x\right)+B_{10} \sinh \left(\omega_{1} x\right) \cos \left(\omega_{1} x\right) \\
& +B_{11} \cosh \left(\omega_{1} x\right) \sin \left(\omega_{1} x\right) \\
& +B_{12} \cosh \left(\omega_{1} x\right) \cos \left(\omega_{1} x\right)
\end{aligned}
$$

The abbreviations used in the expressions above were given in the Appendix. The moment effect is introduced in the formulation (see the Appendix). The superscripts I, II, and III denote the relevant stress components of the three individual regions (see Figure 1), $\sigma_{1 x}$ is the longitudinal normal stress of the adherends. $\sigma_{i y}$ and $\tau_{i x}(i=3,4)$ are the peel and shear stresses along the adhesive midplane. Set of (1a) and (1c) are related to the right and left flexible adhesive regions. Set of (1b) is related to the stiff adhesive region, the central region. Normal, shear, and peel stresses can then be evaluated by 


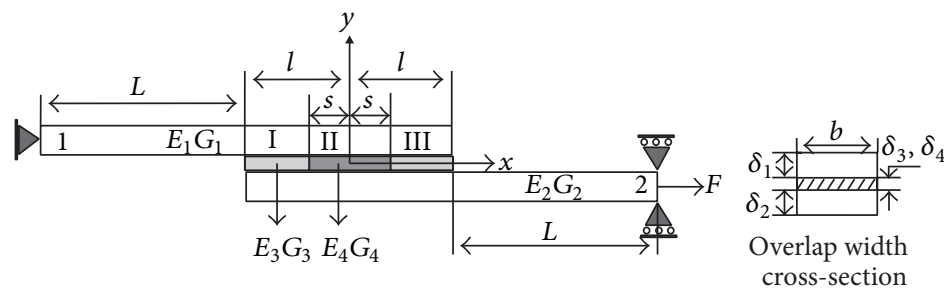

(a)

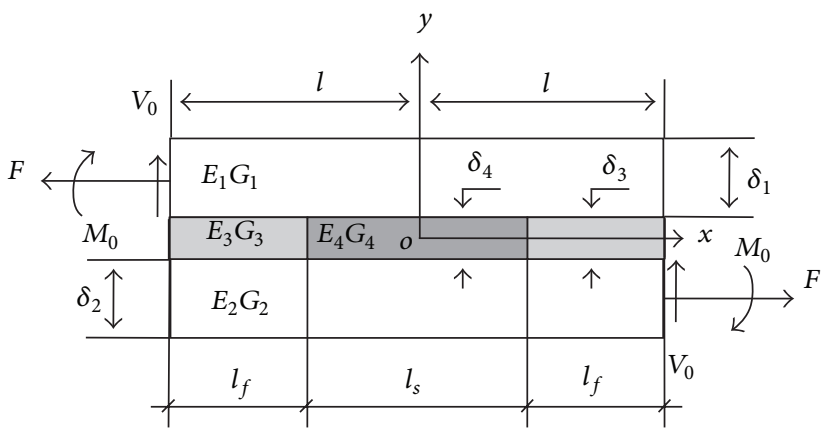

(b)

FIGURE 1: Biadhesive single lap joint under a tensile load: (a) geometric and material parameters, (b) force equilibrium free-body diagram.
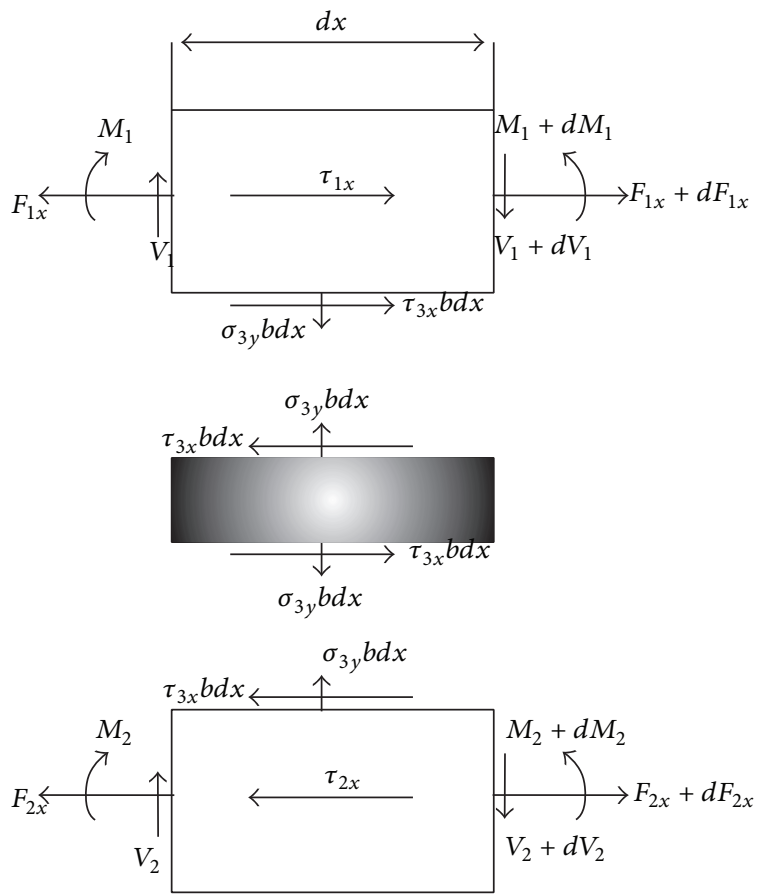

FIGURE 2: Free-body force equilibrium diagram.

using the appropriate expressions for each region. The results of analytical solutions were compared with both the results of 2D and 3D FEM analyses.

\section{Finite Element Model}

Geometry, dimensions, and boundary conditions of the biadhesive single-lap joint are shown in Figure 4.
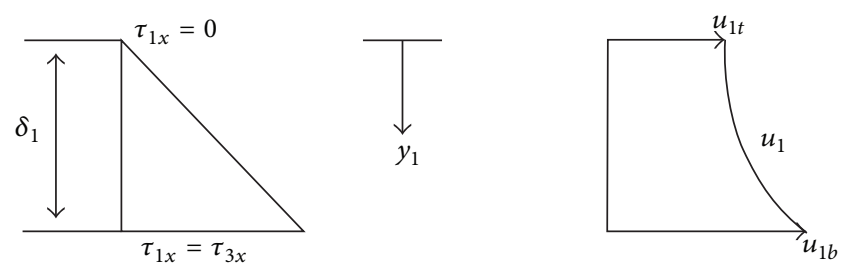

(a)
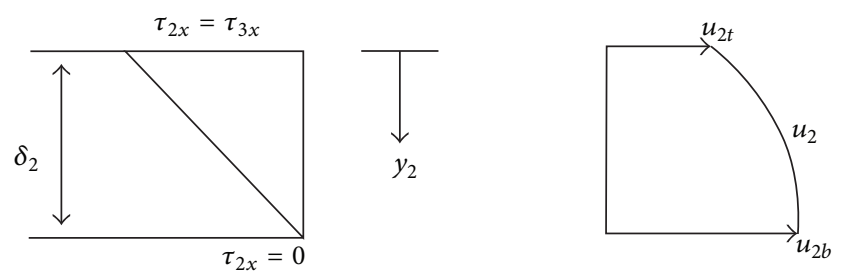

(b)

FIGURE 3: Longitudinal shear stress and displacement distributions through the thickness of the adherends; (a) upper adherend, (b) lower adherend.

Finite Element Analysis based on the surface to surface contact model was performed by using commercial FEA software ANSYS. The adherends were constrained at the ends, preventing excessive deflection of the joint and simulating the gripping in the testing machine. The effect of the gripping length on the stress components was studied to validate the stress analysis results. Convergence tests were carried out by using the gripping lengths of 10,20 , and $30 \mathrm{~mm}$. It was seen that the gripping length does not have a considerable effect on the stress results [29, see page 118]. Finally, it was concluded that the $20 \mathrm{~mm}$ optimum length was enough and sufficient to prevent excessive deflection and allow the free 


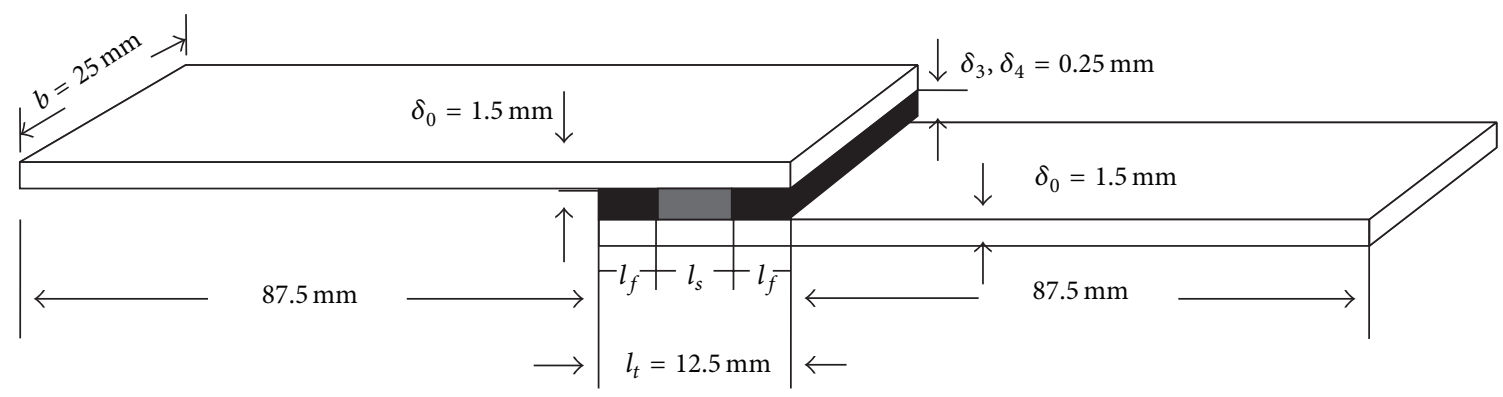

Flexible adhesive

Stiff adhesive

(a)

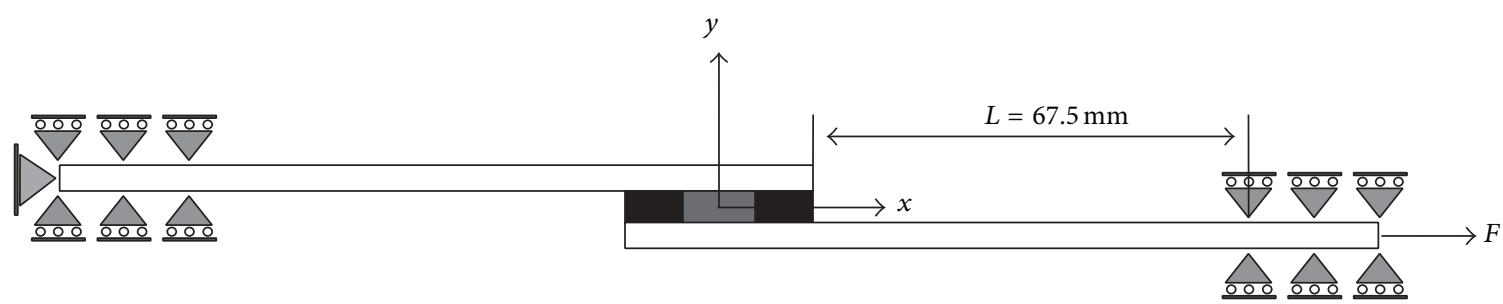

(b)

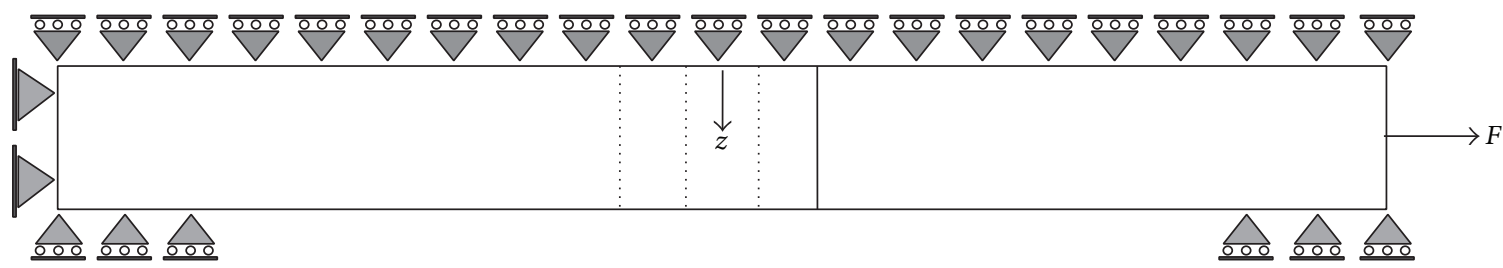

(c)

FIGURE 4: Biadhesive single-lap joint: (a) geometry and dimensions, (b) 2D and 3D FEM model boundary conditions (front view), (c) 3D FEM model boundary conditions (top view).

TABLE 1: Material properties of adherends and adhesives.

\begin{tabular}{lccc}
\hline & $\begin{array}{c}\text { Adherend } \\
\text { (Aluminium alloy 7075) }\end{array}$ & $\begin{array}{c}\text { Flexible Adhesive } \\
\text { (Terokal 5045) }\end{array}$ & $\begin{array}{c}\text { Stiff Adhesive } \\
\text { (Hysol EA 9313) }\end{array}$ \\
\hline Modulus of elasticity $(\mathrm{GPa})$ & $E_{1}=E_{2}=71.700$ & $E_{3}=0.437$ & $E_{4}=2.274$ \\
Modulus of shear $(\mathrm{GPa})$ & $G_{1}=G_{2}=26.955$ & $G_{3}=0.158$ & $G_{4}=0.836$ \\
Poisson's ratio & 0.33 & 0.38 & 0.36 \\
Shear strength $(\mathrm{MPa})$ & 152 & 20 & 27.6 \\
Elongation at break $(\%)$ & 10 & 11.3 & 8 \\
\hline
\end{tabular}

rotation of the joint for the problem considered (Figure 4(b)). Thus, overlap region was not influenced by the boundary conditions. Due to the longitudinal symmetry, half-symmetry boundary condition was used in order to reduce the solution time for the three-dimensional analysis (Figure 4(c)) [30].

Aluminium alloy 7075 was used as identical adherends. Hysol EA 9313 and Terokal 5045 epoxy adhesives, produced by Henkel, were used as stiff and flexible adhesives, respectively. Material properties of adherends and adhesives are given in Table 1 [31].

The thicknesses of the adherends and adhesives are $1.5 \mathrm{~mm}$ and $0.25 \mathrm{~mm}$, respectively. The total overlap length $l_{t}, 2 l_{f}+l_{s}$, was taken as $12.5 \mathrm{~mm}$, where $l_{f}$ is the length of the flexible adhesive and $l_{s}$ is the length of the stiff adhesive. The bond-length ratios of the adhesive bondline varied as $\xi=$ $l_{f} / l_{s}=0.2,0.4,0.7,1.3$. Therefore, four different biadhesive bondline configurations were investigated. A static force of $3.6 \mathrm{kN}$ was applied to the right side of the lower adherend. (Loading was chosen as the same in our recent paper [31]). The finite element models of the joint for the 2D and 3D FEM are shown in the same figure (Figure 5).

The finite element contact model recognizes possible contact pairs by the presence of specific contact elements. The overlap surfaces of the adherends and the adhesives were 


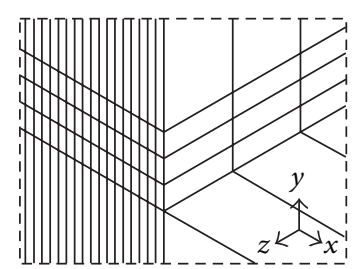

(b)

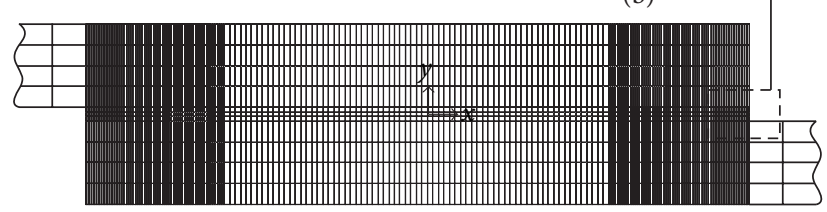

(a)

FIGURE 5: Finite element model of the joint: (a) two-dimensional, (b) three-dimensional.

modelled using surface-to-surface contact for the 2D and 3D finite element models. Surface-to-surface contact model uses Gauss integration points as a default, which generally provide more accurate results than the nodal detection scheme, which uses the nodes themselves as the integration points. This type of contact model transmits contact pressure between Gauss points, and not force between nodes.

In 2D FEA model, contact pairs were created using contact element "CONTA172" and target element "TARGE169" between the adherends and adhesive overlap surfaces (Figure 6). CONTA172 elements were generated on the bottom and upper surfaces of adhesive. CONTA172 is a 2D, 3-node, higher order parabolic element. TARGE169 elements were used on the overlap surfaces of the adherends. TARGE169 is used to represent various 2D "target" surfaces for the associated contact element. The $2 \mathrm{D}$ model was discretized using the 8-node plane stress elements (Plane 183).

In 3D FEA model, contact pairs were created using contact element "CONTA174" and target element "TARGE170" between the adherends and adhesive overlap surfaces (Figure 6). CONTA174 elements were generated on the bottom and upper surfaces of adhesive. CONTA174 is a $3 \mathrm{D}, 8$-node, higher order quadrilateral element. TARGE170 elements were used on the overlap surfaces of the adherends. TARGE170 is used to represent various 3D "target" surfaces for the associated contact element. (More detailed information on the $3 \mathrm{D}$ contact modelling of biadhesive joint is available in our recent paper [31].) Adherends and adhesives were meshed with solid hexahedral elements (solid95). This element is defined by twenty nodes having three degrees of freedom per node. Three elements were used through the thickness of the adherends and the bondline (Figure 5(b)).

The mesh density affects the stress values in the adhesive layer. The size of the elements in the mesh was reduced until a stable stress value was achieved. Five different meshing schemes were investigated to optimize the number and size of the elements. The sizes of the elements were graded for the finer mesh in the critical regions (at the edges of overlap and contact interfaces of adhesives). Finally, the element size near the stress concentration and contact interface were set

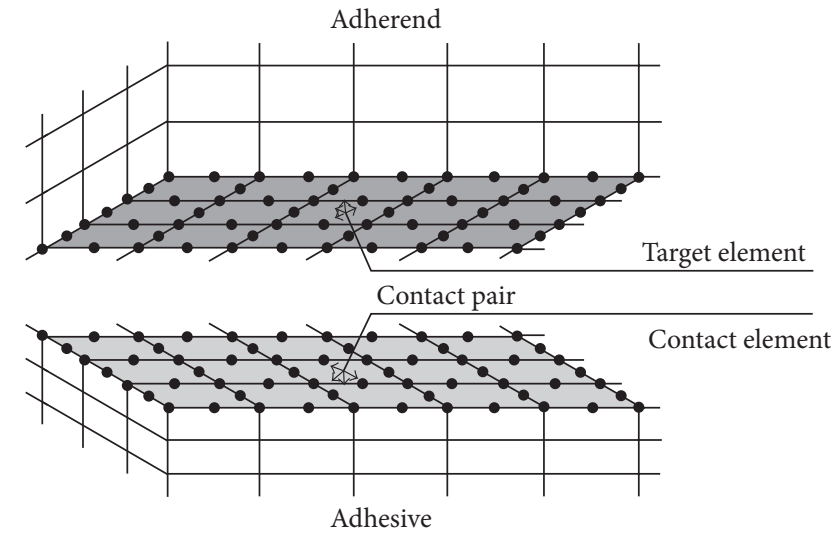

Figure 6: Surface-to-surface contact pair.

to $0.03 \mathrm{~mm}$ and $0.04 \mathrm{~mm}$, respectively, which is fine enough to describe the severe stress variation near the critical regions. The number of elements varied for FEM models of mono- and biadhesive joints. However, the mesh size was kept constant in all models. This was important in order to compare the results between the mono- and biadhesive joints, and also to compare results of biadhesive joints among themselves.

The two-dimensional and three-dimensional finite element analyses were performed. The results were used to compare the analytically predicted adhesive stresses. We present the FEA results and their comparisons with the analytical results in the next section.

\section{Results and Discussion}

The adhesive shear and peel stress distributions in the midplane of the bondline were obtained both analytically and numerically. This section presents a comparison between the numerical and analytical results. The distributions of the stress components were normalized with respect to average shear stress $\left(\tau_{\mathrm{avg}}\right)$, and distributions of the same type of stress components were given in the same figure. In addition, bar 


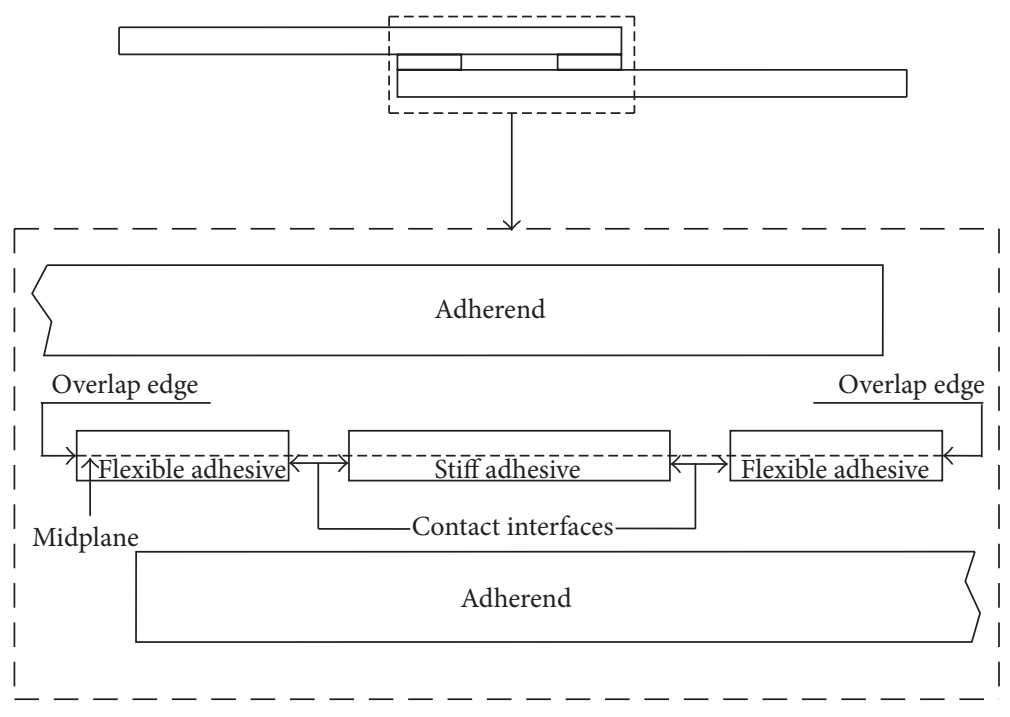

FIGURE 7: Overlap region of the biadhesive single-lap joint.

graphs were used to illustrate a visual comparison among the stress results of analytical model, 2D FEA and 3D FEA.

Some comparisons among the peak stress values were carried out on the basis of the percent error. Percentage error is defined as follows:

$$
\text { Error } \%=\frac{\text { Numeric }- \text { Analytic }}{\text { Numeric }} \times 100
$$

where word "Numeric" denotes 2D and 3D FEA results. Percent errors between analytical and numerical results for the biadhesive case were tabulated in tables, while the error discussions were presented in a text for monoadhesive case.

In the bar graphs and tables, peak values of the stress components obtained through analytical model were compared with those obtained through 2D FEA and 3D FEA. However, there are two peak stress locations for the biadhesive bondline, one just at the overlap edges and the other at contact interfaces of adhesives. Therefore, in the case of biadhesive joint, two peak stresses were considered for the comparison (Figure 7). In 3D FEA, shear and peel stresses peak at the midwidth of the adhesive midplane for both of mono- and biadhesive bondlines. Therefore, stress distributions plotted at the midwidth along the adhesive midplane. Peak values are also related to the midwidth of the adhesive midplane in $3 \mathrm{D}$ FEA.

Figure 8 shows the shear stress distributions of the monoand biadhesive joints obtained from analytical and numerical (FEA) solutions. It can be seen from Figure 8 that the shear stress distributions in the monoadhesive joint is not uniform. This is because the applied load causes bending moments which lead to peel stresses especially at the overlap edges. However, peel stress component is more dominant especially over the mono-stiff adhesive. Therefore, the shear stress distribution for the mono-flexible adhesive is more uniform and there is a very small stress concentration at the overlap edges. In the monoadhesive joint, the higher level of stress region exists at the overlap edges (Figure 8).
In the biadhesive bondline, as can be seen in Figure 8, higher shear stress occurs at the contact interfaces of the adhesives, and the lower shear stress region exists at the overlap edges. Peak stress decreases at the overlap edges and increases at the contact interfaces. With the increase in the $\xi$ ratio, the values of peak shear stress are decreasing, although there is no great reduction in the peak shear stress for ratio higher than 0.7 .

The shear stress distributions from the analytical solution were plotted in Figure 8(c). This shows a good agreement with numerical ones, although some differences can be seen at the overlap edges. Peak shear stresses of the numerical solutions occur very near the edge of the overlap region, while those of the analytical results were always at the overlap edges. That is because the numerical model tries to model the zero stress state at traction-free end surfaces. However, in practice, there is an adhesive spew fillet and the shear stress does not go to zero at the bondline ends.

The normalized peak shear stress values at the overlap edges and the contact interfaces are showed in Figure 9 as a vertical bar chart.

From Figure 9, it can be seen that the normalized peak shear stress values for analytical and the numerical models had close correlation for the majority of mono- and biadhesive joints. The maximum shear stresses occurred at the overlap edges for monoadhesive joints, and at the contact interfaces for biadhesive joints.

According to Figure 9, the shear peak stress which occurs at the overlap edges increased with increasing $\xi$ ratio. Furthermore, increasing rate in the shear peak stress at the edges was observed to be a parallel changing rate with the decreasing rate in the shear peak stress at the contact interfaces.

Figures 9(a) and 9(b) show that analytical results are in good agreement with 2D FEA results rather than 3D FEA results for both the mono- and biadhesive joint cases. The 3D FEA solution gives slightly higher shear peak stress values than those from the analytical and 2D FEA solutions. 


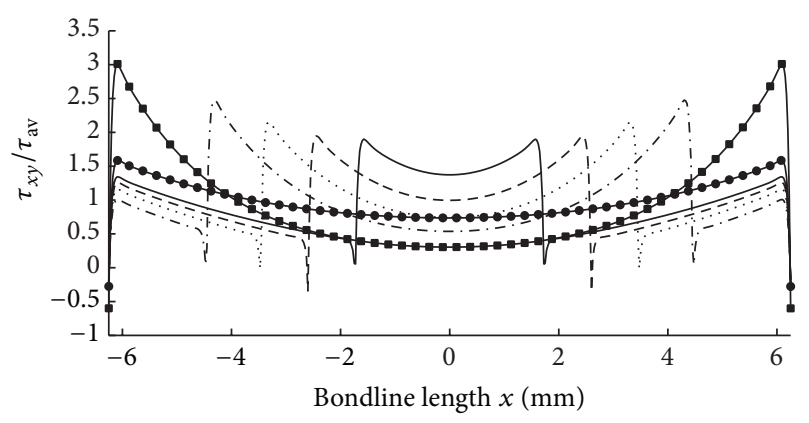

(a)

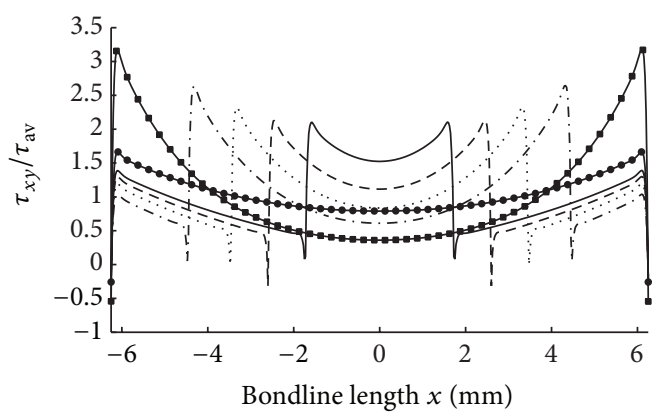

(b)

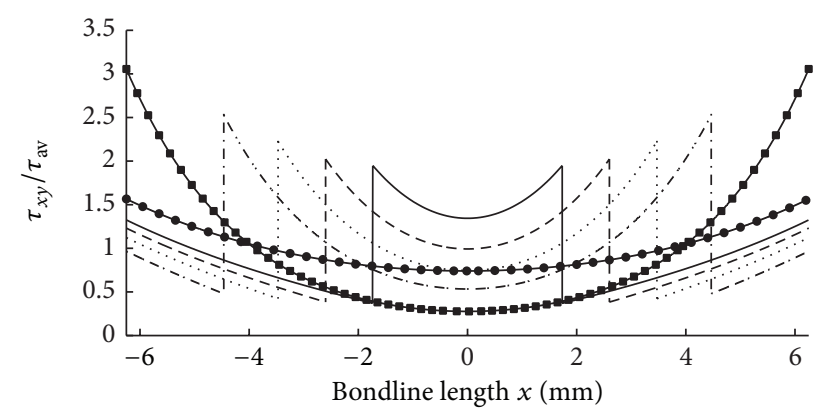

$\rightarrow$ Stiff adhesive
$\rightarrow$ Flexible adhesive
$\longrightarrow \xi=1.3$

- - $\xi=0.7$

…. $\xi=0.4$

... $\xi=0.2$

(c)

FIGURE 8: (a) Normalized shear stress distributions obtained from 2D FEA. (b) Normalized shear stress distributions at midwidth obtained from 3D FEA. (c) Normalized shear stress distributions obtained from analytical model.

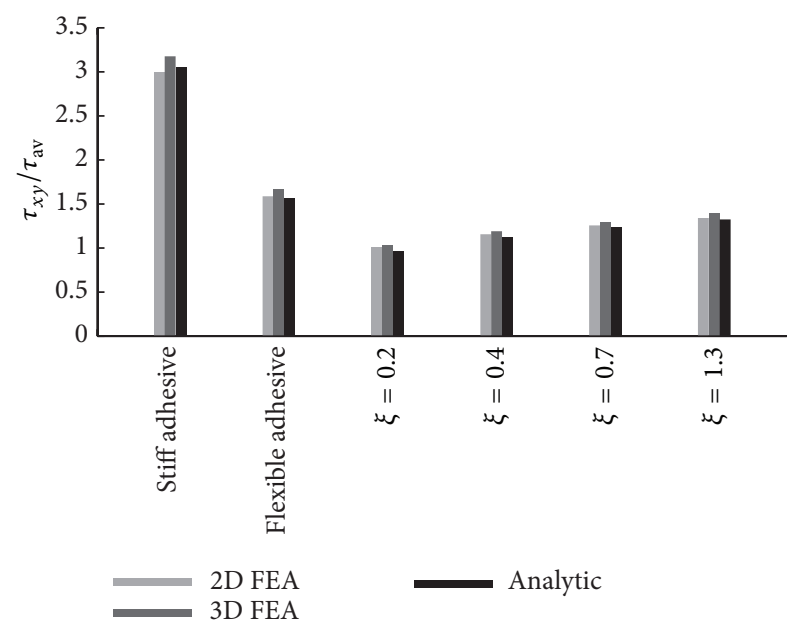

(a)

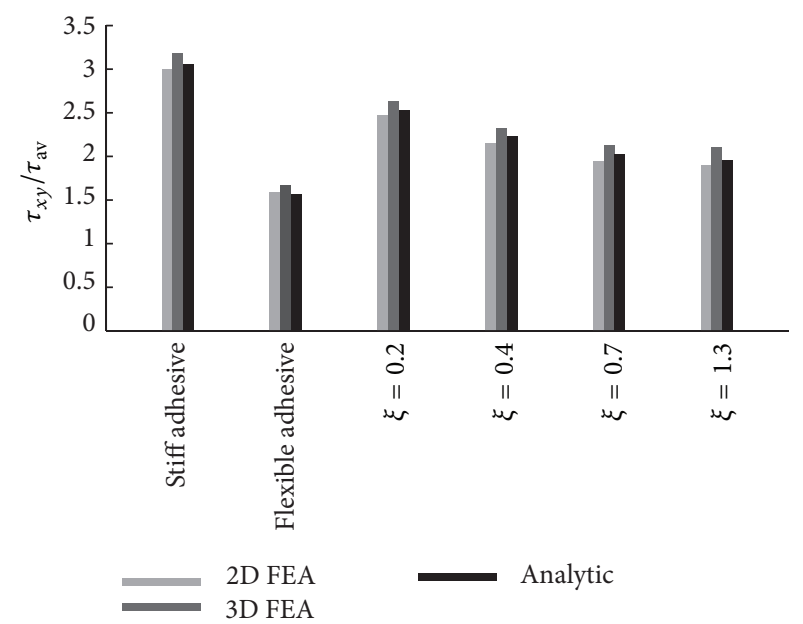

(b)

FIgURE 9: (a) Normalized peak shear stress values at the overlap edges. (b) Normalized peak shear stress values at the contact interfaces.

As stated before, percent error comparisons for monoadhesive joint will be given in a text. After doing some calculations by data used to plot Figure 9, it was concluded that, for mono-stiff adhesive joint, the analytical model predicts higher peak shear stress than the 2D FEA with an error of at most $2.0 \%$, while it predicts a lower peak shear stress than $3 \mathrm{D}$
FEA with an error of at most $3.80 \%$. However, for the monoflexible adhesive bondline, analytical model predicts lower peak shear stress than both the 2D FEA and 3D FEA, with errors of $1.40 \%$ and $5.94 \%$, respectively. For more detailed comparison about the biadhesive joint, percent errors were calculated by using (2) and tabulated in Table 2. 


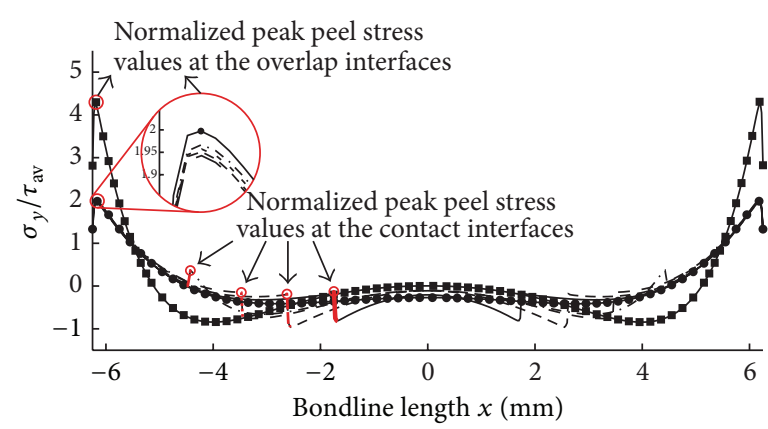

(a)

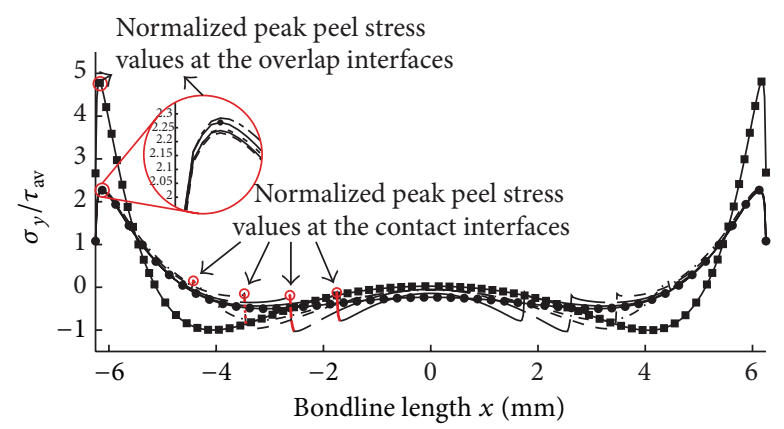

(b)

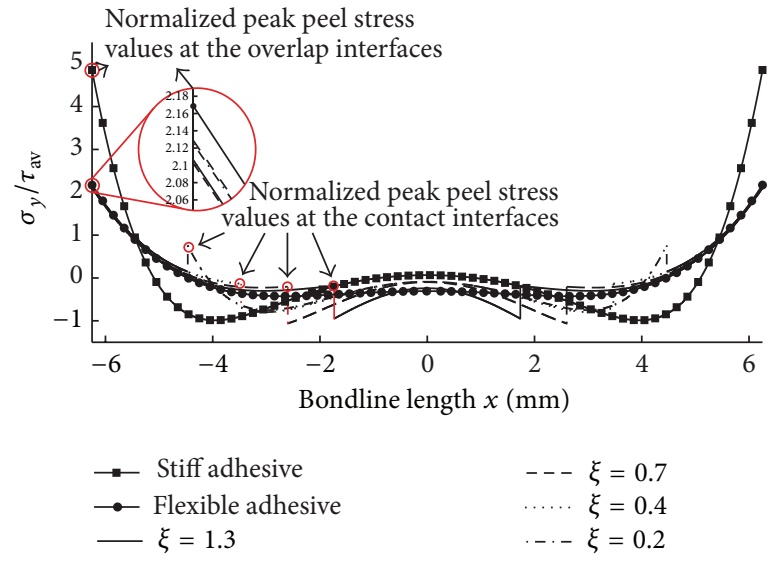

(c)

FIGURE 10: (a) Normalized peel stress distributions obtained from 2D FEA. (b) Normalized peel stress distributions at the midwidth obtained from 3D FEA. (c) Normalized peel stress distributions obtained from analytical model.

TABLE 2: Percent error between analytical and numerical results for the peak shear stresses.

\begin{tabular}{ccc}
\hline \multicolumn{3}{c}{ Percent error (\%) } \\
& At the contact interfaces & At the overlap edges \\
\hline 2-D FEA & & \\
$\xi=0.2$ & -2.14 & 3.93 \\
$\xi=0.4$ & -3.62 & 3.02 \\
$\xi=0.7$ & -4.11 & 2.06 \\
$\xi=1.3$ & -2.57 & 1.19 \\
3 -D FEA & & \\
$\xi=0.2$ & 3.98 & 6.62 \\
$\xi=0.4$ & 4 & 5.79 \\
$\xi=0.7$ & 4.84 & 5.08 \\
$\xi=1.3$ & 7.32 & 4.60 \\
\hline
\end{tabular}

Peak shear stress values were given at the adhesive contact interface and overlap edge for the four bond-length ratios. As can be seen from Table 2, the analytical results agree well with the numerical ones. It can be calculated form the data in Table 2 that, at the overlap edges, peak shear stresses predicted by the analytical model agree well with the 2D FEA results with a maximum error of about $3.93 \%$ compared to that of $6.62 \%$ for the $3 \mathrm{D}$ FEA results. The analytical results have a high level of agreement with the 2D FEA results at the contact interfaces of adhesives. The maximum percent error is minus $4.11 \%$. If $3 \mathrm{D}$ FEA results were considered, there was a lower level of agreement between analytical and 3D FEA results with a maximum error of $7.32 \%$.

Figure 10 shows the peel stress distributions of the monoand biadhesive joints obtained from analytical and numerical solutions. It is clear from Figure 10 that the peel stress peaks at the overlap edges for both mono- and biadhesive joints. The biadhesive bondline gives lower peak peel stresses at the overlap edges. However, the peak peel stress in the flexible part of the biadhesive bondline is approximately within the same order of magnitude for both mono- flexible and biadhesive joints.

From Figure 10, it must be noted that, at the overlap edges, changing the $\xi$ ratio has a moderating effect on the values of the peel peak stresses. The peel peak stress in the biadhesive bondline was lower than that of the mono-stiff adhesive bondline. At the contact interfaces of adhesives, it can be seen from Figure 10 that the presence of a little amount of flexible adhesive can cause a secondary peak in the peel stress distribution in the biadhesive joint.

Figure 11 shows a comparison as a vertical bar chart of the normalized peel peak stresses obtained from analytical and numerical solutions.

It can be seen from Figure 11 that the analytical and numerical peel peak stresses have close correlation for 


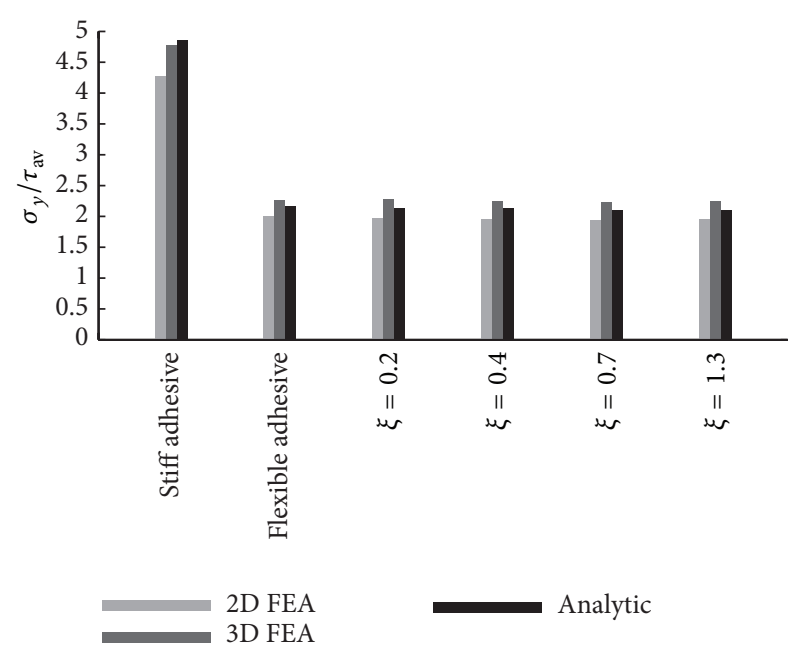

(a)

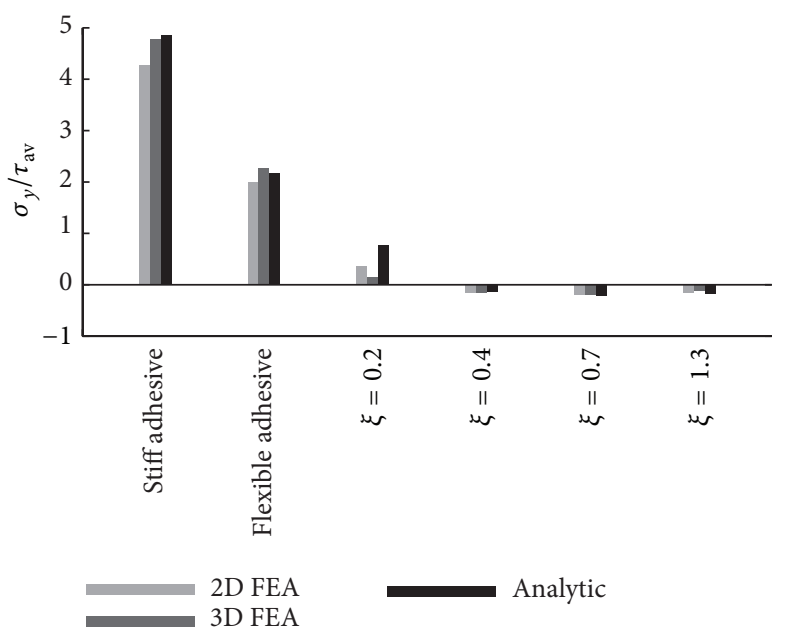

(b)

FIGURE 11: (a) Normalized peak peel stress values at the overlap edges. (b) Normalized peak peel stress values at the contact interfaces.

the majority of mono- and biadhesive joints, especially at the overlap edges. The peak values occurred at the overlap edges for both the mono- and biadhesive joints. Figure 11(a) shows that, at the mono- and biadhesive overlap edges, the analytical results are in a good agreement with the $3 \mathrm{D}$ FEA results. For mono-stiff adhesive joint, the analytical model predicts higher peel peak stress than both the $2 \mathrm{D}$ FEA and 3D FEA results, with an error of $-13.62 \%$ and $-1.48 \%$, respectively. However, for the mono-flexible adhesive bondline, analytical model predicts higher peel peak stress than the 2D FEA with an error of $-8.55 \%$, while it predicts lower peel peak stress than 3D FEA results, with an error of $4.40 \%$. Apparent difference about the results for the monostiff adhesive joint is that analytical results show a higher peak peel stress compared to the peak values of the 3D FEA results (Figure 11(a)). The largest difference in the peak values occurred at the contact interfaces of adhesives, when the $\xi$ ratio is set to 0.2 . This difference is not very important because, in order to design an adhesively bonded joint, the values of the maximum peak stresses are more important than the exact stress distribution [32].

For more detailed comparison about the biadhesive joint, peak peel stress values were tabulated in Table 3. Table 3 compares the analytical results with numerical results for peel peak stresses of biadhesive joint.

As seen in Table 3, a good correlation was found between the analytical and numerical results at the overlap edges. The maximum error between the analytical and 2D FEA results is $-9.12 \%$. However, if $3 \mathrm{D}$ FEA results are considered, there is a higher level of agreement between analytical and $3 \mathrm{D}$ FEA results with a maximum error of $7 \%$. The maximum error in the peak values occurred at the contact interfaces of adhesives. The magnitude of the error was maximum for the bond-length ratio of 0.2 . For this ratio, analytical model predicts higher peel peak stress than both the 2D FEA with an error of $-111.46 \%$ and $3-D$ FEA results with an error of $-408.86 \%$.
TABLE 3: Percent error between analytical and numerical results for the peel peak stresses.

\begin{tabular}{rcc}
\hline & \multicolumn{2}{c}{ Percent error (\%) } \\
& At the contact interfaces & At the overlap edges \\
\hline 2 -D FEA & & \\
$\xi=0.2$ & -111.46 & -8.03 \\
$\xi=0.4$ & 11.41 & -9.12 \\
$\xi=0.7$ & -10.57 & -8.18 \\
$\xi=1.3$ & -24.57 & -7.94 \\
3 -D FEA & & \\
$\xi=0.2$ & -408.86 & 7 \\
$\xi=0.4$ & 10.87 & 5.16 \\
$\xi=0.7$ & -8.28 & 5.78 \\
$\xi=1.3$ & -48.85 & 5.98 \\
\hline
\end{tabular}

\section{Conclusions}

Some expressions were derived related to stress components for the single lap joint with biadhesive bondlines by following the same steps as Zhao's solutions. However, Zhao compared stress results predicted by the closed-form expressions only with the 2D FEA results. Moreover, the bond-length ratio for the biadhesive layer stayed constant in his study.

In this study, the analytical formulations were presented in a detailed format (including further details) and given in the Appendix. A MAPLE program was written. Prepared MAPLE program employs these expressions to calculate stress components. Some figures were plotted using the data calculated with MAPLE. The closed-form solution accounts the bending effect. It is capable of determining the distribution of the stress components only in the middle of bondline. (It must be noted that stress expressions are only a function of $x$. As seen in Figure 4 , the $x$-axis passes through the midplane of the adhesive layer.) 
The validity of the analytical results was assessed by comparing the analytical results with the $2 \mathrm{D}$ and $3 \mathrm{D}$ FEA results. Especially, the adhesive peel stress is sensitive to the three-dimensional effects. Therefore, the three-dimensional FEM model can provide more accurate predictions for comparing the results. In this study, the $2 \mathrm{D}$ and $3 \mathrm{D}$ FEM models were based on the surface-to-surface contact elements. Analyses were performed for four different bond-length ratios both analytically and numerically. In addition, a comparison of peak stress values was carried out on the basis of percentage error. results.

The following conclusions can be made about the analysis

(1) The analytical model gives similar stress distributions to those of numerical models for all the stress components.

(2) The peak stress comparison shows that analytical results are generally in a good agreement with the numerical ones. The analytical predictions are more compatible with 3D FEA predictions for the peel peak stresses in the case of the biadhesive bondline. As stated above, the adhesive peel stress is sensitive to the three-dimensional effects. Thus, as to its peel peak stress predictions, it can be said that the analytical model has somewhat more ability to model the 3D effects than 2D FEM model. However, for the shear peak stresses, the analytical model gives closer results to those of the 2D (plane stress) FEA. These comparisons are valid for both the mono- and biadhesive joints. Zhao [28] reported in his paper that the peak shear stresses at the overlap edges predicted by $2 \mathrm{D}$ FEA were lower than those predicted by the analytical solution. Our study offers opposite case. This can be attributed to the differences in FEM models and mesh concentrations. In our FEM model, the surface-tosurface contact model was used. In addition, there was also a difference in the predicted peak locations. Zhao concluded that shear peak stresses of the 2D FEA results occurred at about one bondline thickness away from the ends, while those of the analytical results were always at the ends of the overlap region. However, in this study, peak shear stress occurs at a point closer to the ends, approximately $70 \%$ of the bondline thickness.

(3) The analytical and numerical results show that appropriate $\xi$ ratio must be used to effectively reduce the concentration of peel and shear stresses in the joint.

(4) Choosing improper bond-length ratio, using insufficient amount of flexible adhesive in the biadhesive bondline such as $\xi=0.2$ in our model, caused a secondary peak in the peel stress distribution because of the change of peel stresses from compressive to tensile. Therefore, selecting the appropriate bondlength ratio is crucial for biadhesive joints.

(5) The results show a measurable decrease in the stress components of the biadhesive joints compared with those in which monoadhesives were used over the full length of the bondline.

\section{Appendix}

The detailed procedure for deriving stress components expressions for the biadhesive single-lap joint was presented below and given by Zhao [28]. Firstly, we begin with the free-body diagram of the flexible adhesive layer given in Figure 2. By referring to Figure 2, the differential equations of equilibrium equations can be obtained as

$$
\begin{aligned}
\frac{d F_{1 x}}{d x}+\tau_{3 x} b & =0, & & \frac{d F_{2 x}}{d x}-\tau_{3 x} b=0, \\
\frac{d V_{1}}{d x}-\sigma_{3 y} b & =0, & & \frac{d V_{2}}{d x}+\sigma_{3 y} b=0,
\end{aligned}
$$

Based upon the assumptions (see Figure 3), one can write the following stress-strain relationships:

$$
\begin{gathered}
\sigma_{1 x}=E_{1} \varepsilon_{1 x}=E_{1} \frac{d u_{1}}{d x}, \\
\sigma_{2 x}=E_{2} \varepsilon_{2 x}=E_{2} \frac{d u_{2}}{d x}, \\
\sigma_{3 y}=E_{3} \varepsilon_{3 y}, \\
\gamma_{1 x}=\frac{\tau_{1 x}}{G_{1}}, \quad \gamma_{2 x}=\frac{\tau_{2 x}}{G_{2}}, \quad \gamma_{3 x}=\frac{\tau_{3 x}}{G_{3}} .
\end{gathered}
$$

From the equilibrium of $x$ forces in Figure 2, we have

$$
F=F_{1 x}+F_{2 x} .
$$

The stress distributions through the thickness of adherends are assumed to behave linearly (Figure 3). Then, the shear stresses acting on upper and lower adherends can be expressed as

$$
\begin{aligned}
\tau_{1 x}=\tau_{3 x} \frac{y_{1}}{\delta_{1}}, & 0 \leq y_{1} \leq \delta_{1}, \\
\tau_{2 x}=\tau_{3 x}\left(1-\frac{y_{2}}{\delta_{2}}\right), & 0 \leq y_{2} \leq \delta_{2},
\end{aligned}
$$

where $y_{1}$ and $y_{2}$ are the distances from the top of adherend 1 and adherend 2, respectively. Substituting (A.6a) and (A.6b) into (A.4b), we obtain the shear strains of adherends as follows:

$$
\gamma_{1 x}=\frac{\tau_{3 x}}{\delta_{1}} \frac{y_{1}}{G_{1}}, \quad \gamma_{2 x}=\frac{\tau_{3 x}}{G_{1}}\left(1-\frac{y_{2}}{\delta_{2}}\right) .
$$


Longitudinal displacements of adherend 1 and 2 due to the longitudinal forces are given as follows:

$$
\begin{gathered}
u_{1}^{T}\left(x, y_{1}\right)=u_{1 t}^{T}+\int_{0}^{y_{1}} \gamma_{1 x} d \widehat{y_{1}}=u_{1 t}^{T}+\frac{\tau_{3 x} y_{1}^{2}}{2 G_{1} \delta_{1}}, \\
u_{2}^{T}\left(x, y_{2}\right)=u_{2 t}^{T}+\int_{0}^{y_{2}} \gamma_{2 x} d \widehat{y_{2}}=u_{2 t}^{T}+\frac{\tau_{3 x}}{G_{2}}\left(y_{2}-\frac{y_{2}^{2}}{2 \delta_{2}}\right),
\end{gathered}
$$

where subscripts $1 t$ and $2 t$ correspond to the top surfaces of adherend 1 and adherend 2, respectively. Superscript $T$ was used for emphasizing the longitudinal force effects, which serves to distinguish it from the moment effects. The moment effect was also introduced in the later steps of formulation. As an example, we can try to write an expression for the displacement at the interface between adherend 1 and adhesive by using (A.8a) as follows:

$$
u_{1 b}^{T}=u_{1}^{T}\left(y_{1}=\delta_{1}\right)=u_{1 t}^{T}+\frac{\tau_{3 x} \delta_{1}}{2 G_{1}},
$$

where subscript $1 b$ corresponds to the top surface of adherend 1. Using (A.9), we can rewrite (A.8a) as

$$
u_{1}^{T}\left(x, y_{1}\right)=u_{1 b}^{T}-\frac{\tau_{3 x} \delta_{1}}{2 G_{1}}+\frac{\tau_{3 x} y_{1}^{2}}{2 G_{1} \delta_{1}}
$$

Taking (A.8a) and (A.8b) into account, longitudinal force components can be written as

$$
\begin{aligned}
F_{1 x} & =\int_{0}^{\delta_{1}} \sigma_{1 x}^{T}\left(y_{1}\right) b d y_{1} \\
& =E_{1} \int_{0}^{\delta_{1}} \frac{d u_{1}^{T}}{d x}\left(y_{1}\right) b d y_{1} \\
& =E_{1} b \delta_{1}\left[\frac{d u_{1 b}^{T}}{d x}-\frac{\delta_{1}}{3 G_{1}} \frac{d \tau_{3 x}}{d x}\right], \\
F_{2 x} & =\int_{0}^{\delta_{2}} \sigma_{2 x}^{T}\left(y_{2}\right) b d y_{2} \\
& =E_{2} \int_{0}^{\delta_{2}} \frac{d u_{2}^{T}}{d x}\left(y_{2}\right) b d y_{2} \\
& =E_{2} b \delta_{2}\left[\frac{d u_{2 t}^{T}}{d x}+\frac{\delta_{2}}{3 G_{2}} \frac{d \tau_{3 x}}{d x}\right] .
\end{aligned}
$$

From Figure 3, shear strain in the low modulus adhesive is

$$
\gamma_{3 x}=\frac{u_{2 t}-u_{1 b}}{\delta_{3}}
$$

Substituting (A.12) into (A.4b) and then differentiating it with respect to $x$, we obtain

$$
\frac{\delta_{3}}{G_{3}} \frac{d \tau_{3 x}}{d x}=\left[\frac{d u_{2 t}}{d x}-\frac{d u_{1 b}}{d x}\right] .
$$

To get the displacements induced by the bending moment, we can rearrange $u_{1 b}$ and $u_{2 t}$ displacements in terms of the tensile and moment effects in the following form:

$$
u_{1 b}=u_{1 b}^{T}-u_{1 b}^{M}, \quad u_{2 t}=u_{2 t}^{T}+u_{2 t}^{M} .
$$

Longitudinal strains may now be written in terms of bending moments by using classical beam theory as

$$
\frac{d u_{1}^{M}}{d x}=\frac{M_{1} \delta_{1}}{E_{1} I_{1} 2}=\frac{6 M_{1}}{E_{1} b \delta_{1}^{2}}, \quad \frac{d u_{2}^{M}}{d x}=\frac{M_{2} \delta_{2}}{E_{2} I_{2} 2}=\frac{6 M_{2}}{E_{2} b \delta_{2}^{2}} .
$$

Differentiating (A.14) with respect to $x$ and substituting it and (A.15) into (A.13), we have

$$
\frac{\delta_{3}}{G_{3}} \frac{\tau_{3 x}}{d x}=\frac{d u_{2 t}^{T}}{d x}-\frac{d u_{1 b}^{T}}{d x}+\frac{6 M_{1}}{E_{1} b \delta_{1}^{2}}+\frac{6 M_{2}}{E_{2} b \delta_{2}^{2}} .
$$

Substituting (A.15) into (A.11a) and (A.11b) and after simplifying the expression, we get

$$
\begin{aligned}
{\left[\frac{\delta_{3}}{G_{3}}\right.} & \left.+\frac{\delta_{1}}{3 G_{1}}+\frac{\delta_{2}}{3 G_{2}}\right] \frac{d \tau_{3 x}}{d x} \\
& =\frac{F_{2 x}}{E_{2} \delta_{2} b}-\frac{F_{1 x}}{E_{1} b \delta_{1}}+\frac{6 M_{1}}{E_{1} b \delta_{1}^{2}}+\frac{6 M_{2}}{E_{2} b \delta_{2}^{2}} .
\end{aligned}
$$

Differentiating (A.17) with respect to $x$ and combining this expression with (A.1) and (A.3), we obtain

$$
\begin{aligned}
{\left[\frac{\delta_{3}}{G_{3}}\right.} & \left.+\frac{\delta_{1}}{3 G_{1}}+\frac{\delta_{2}}{3 G_{2}}\right] \frac{d^{2} \tau_{3 x}}{d x^{2}}-4\left(\frac{1}{E_{2} \delta_{2}}+\frac{1}{E_{1} \delta_{1}}\right) \tau_{3 x} \\
& =\frac{6 V_{1}}{E_{1} b \delta_{1}^{2}}+\frac{6 V_{2}}{E_{2} b \delta_{2}^{2}} .
\end{aligned}
$$

Rearranging (A.18) with (A.1), (A.5) and (A.17) yields

$$
\begin{gathered}
{\left[\frac{\delta_{3}}{G_{3}}+\frac{\delta_{1}}{3 G_{1}}+\frac{\delta_{2}}{3 G_{2}}\right] \frac{d^{2} F_{1 x}}{d x^{2}}-\left(\frac{1}{E_{2} \delta_{2}}+\frac{1}{E_{1} \delta_{1}}\right) F_{1 x}} \\
+\frac{6 M_{1}}{E_{1} \delta_{1}^{2}}+\frac{6 M_{2}}{E_{2} \delta_{2}^{2}}+\frac{F}{E_{2} \delta_{2}}=0 .
\end{gathered}
$$

Differentiating (A.19) twice with respect to $x$ and recalling (A.1), (A.2), and (A.3) and rearranging give us

$$
\begin{gathered}
{\left[\frac{\delta_{3}}{G_{3}}+\frac{\delta_{1}}{3 G_{1}}+\frac{\delta_{2}}{3 G_{2}}\right] \frac{d^{4} F_{1 x}}{d x^{2}}-4\left(\frac{1}{E_{1} \delta_{1}}+\frac{1}{E_{2} \delta_{2}}\right) \frac{d^{2} F_{1 x}}{d x^{2}}} \\
+6 b\left(\frac{1}{E_{1} \delta_{1}^{2}}-\frac{1}{E_{2} \delta_{2}^{2}}\right) \sigma_{3 y}=0 .
\end{gathered}
$$

Curvatures of adherends 1 and 2 are

$$
\frac{d^{2} v_{1}}{d x^{2}}=-\frac{M_{1}}{E_{1} I_{1}}, \quad \frac{d^{2} v_{2}}{d x^{2}}=-\frac{M_{2}}{E_{2} I_{2}},
$$


where $v_{1}$ and $v_{2}$ are the transverse displacements of adherends. $I_{1}$ and $I_{2}$ are the second moments of areas for adherends 1 and 2 and defined as $I_{1}=b \delta_{1}^{3} / 12$ and $I_{2}=$ $b \delta_{2}^{3} / 12$. The interfacial peel stress-strain relationship may be written in terms of the transverse deflections of adherends as

$$
\frac{\sigma_{3 y}}{E_{3}}=\varepsilon_{3 y}=\frac{\nu_{1}-v_{2}}{\delta_{3}} \Longrightarrow \frac{\delta_{3}}{E_{3}} \sigma_{3 y}=v_{1}-v_{2} .
$$

Differentiating the second expression of (A.22) with respect to $x$ yields

$$
\frac{\delta_{3}}{E_{3}} \frac{d \sigma_{3 y}}{d x}=\frac{d \nu_{1}}{d x}-\frac{d \nu_{2}}{d x}=\theta_{1}-\theta_{2},
$$

where $\theta_{1}$ and $\theta_{2}$ are the relevant slopes of adherends. Differentiating (A.23) with respect to $x$ and introducing (A.21) lead to

$$
\frac{\delta_{3}}{E_{3}} \frac{d^{2} \sigma_{3 y}}{d x^{2}}=\frac{12 M_{2}}{E_{2} b \delta_{2}^{3}}-\frac{12 M_{1}}{E_{1} b \delta_{1}^{3}} .
$$

Differentiating (A.24) with respect to $x$ and rearranging yield

$$
\frac{\delta_{3}}{E_{3}} \frac{d^{3} \sigma_{3 y}}{d x^{3}}=\frac{d M_{2}}{d x} \frac{12}{E_{2} b \delta_{2}^{3}}-\frac{d M_{1}}{d x} \frac{12}{E_{1} b \delta_{1}^{3}} .
$$

Substituting (A.3) into (A.25), we obtain

$$
\frac{\delta_{3}}{E_{3}} \frac{d^{3} \sigma_{3 y}}{d x^{3}}=\frac{12 V_{2}}{E_{2} b \delta_{2}^{3}}-\frac{12 V_{1}}{E_{1} b \delta_{1}^{3}}+6 \tau_{3 x}\left(\frac{1}{E_{2} \delta_{2}^{2}}-\frac{1}{E_{1} \delta_{1}^{2}}\right)
$$

Differentiating (A.26) with respect to $x$ and using (A.1) and (A.2) give us

$$
\begin{aligned}
\frac{\delta_{3}}{E_{3}} \frac{d^{4} \sigma_{3 y}}{d x^{4}}= & \frac{d V_{2}}{d x} \frac{12}{E_{2} b \delta_{2}^{3}}-\frac{d V_{1}}{d x} \frac{12}{E_{1} b \delta_{1}^{3}} \\
& +6 \frac{d \tau_{3 x}}{d x}\left(\frac{1}{E_{2} \delta_{2}^{2}}-\frac{1}{E_{1} \delta_{1}^{2}}\right) .
\end{aligned}
$$

Substituting (A.1) and (A.2) into (A.27), we have

$$
\begin{aligned}
\frac{\delta_{3}}{E_{3}} \frac{d^{4} \sigma_{3 y}}{d x^{4}} & +\left(\frac{12}{E_{2} \delta_{2}^{3}}+\frac{12}{E_{1} \delta_{1}^{3}}\right) \sigma_{3 y} \\
& -\frac{6}{b}\left(\frac{1}{E_{1} \delta_{1}^{2}}-\frac{1}{E_{2} \delta_{2}^{2}}\right) \frac{d^{2} F_{1 x}}{d x}=0 .
\end{aligned}
$$

When the same geometric characteristics and material properties are used for the adherends, that is, identical adherends, that allows the straightforward stress analysis of the biadhesive joint. That is,

$$
G_{1}=G_{2}=G, \quad E_{1}=E_{2}=E, \quad \delta_{1}=\delta_{2}=\delta_{0} .
$$

Rearranging (A.20) gives us the below differential equation

$$
\left[\frac{\delta_{3}}{G_{3}}+\frac{2 \delta_{0}}{3 G}\right] \frac{d^{4} F_{1 x}}{d x^{2}}-\frac{8}{E \delta_{0}} \frac{d^{2} F_{1 x}}{d x^{2}}=0 .
$$

Rearranging this equation gives

$$
\frac{d^{4} F_{1 x}}{d x^{4}}-\lambda_{1}^{2} \frac{d^{2} F_{1 x}}{d x^{4}}=0,
$$

where $\lambda_{1}^{2}$ is

$$
\lambda_{1}^{2}=\frac{4 \alpha}{\eta+\beta / 3} .
$$

Here, $\alpha=2 / E \delta_{0}, \eta=\delta_{3} / G_{3}$, and $\beta=2 \delta_{0} / G$. By treating the stiff adhesive layer as the flexible adhesive layer, then $\lambda_{2}^{2}$ becomes

$$
\lambda_{2}^{2}=\frac{4 \alpha}{\eta^{\prime}+\beta / 3},
$$

where $\alpha=2 / E \delta_{0}, \eta=\delta_{3} / G_{4}$ and $\beta=2 \delta_{0} / G$. Rearranging (A.28) by considering (A.29) leads to differential equation given below

$$
\frac{d^{4} \sigma_{3 y}}{d x^{4}}+4 \omega_{1}^{4} \sigma_{3 y}=0
$$

where $\omega_{1}^{4}=3 \phi / \chi$. Here, $\phi=2 / E \delta_{0}^{3}$ and $\chi=\delta_{3} / E_{3}$. By treating the stiff adhesive layer as the flexible adhesive layer, then $\omega_{1}^{4}$ becomes $\omega_{2}^{4}=3 \phi / \chi$. Here, $\phi=2 / E \delta_{0}^{3}$ and $\chi=\delta_{3} / E_{4}$. The governing equations (A.31) and (A.34) are the forth-order ordinary differential equations with constant coefficients. The general solution of (A.31) is

$$
F_{1 x}=A^{\prime} \sinh \left(\lambda_{1} x\right)+B^{\prime} \cosh \left(\lambda_{1} x\right)+C^{\prime} x+D^{\prime},
$$

where $A^{\prime}, B^{\prime}, C^{\prime}$, and $D^{\prime}$ are constants of integration. Then, the longitudinal normal stress can be written as

$$
\sigma_{1 x}=\frac{F_{1 x}}{b \delta_{0}}=A^{\prime \prime} \sinh \left(\lambda_{1} x\right)+B^{\prime \prime} \cosh \left(\lambda_{1} x\right)+C^{\prime \prime} x+D^{\prime \prime},
$$

where $A^{\prime \prime}, B^{\prime \prime}, C^{\prime \prime}$, and $D^{\prime \prime}$ are also constants of integration. The general solution of (A.34) is

$$
\begin{aligned}
\sigma_{3 y}= & A \sinh \left(\omega_{1} x\right) \sin \left(\omega_{1} x\right)+B \cosh \left(\omega_{1} x\right) \sin \left(\omega_{1} x\right) \\
& +C \sinh \left(\omega_{1} x\right) \cos \left(\omega_{1} x\right)+D \cosh \left(\omega_{1} x\right) \cos \left(\omega_{1} x\right) .
\end{aligned}
$$

Using the first expression of (A.1) and the derivative expression of (A.35a) with respect to $x$, the shear stress at the interface then becomes

$$
\tau_{3 x}=-\delta_{1}\left(A^{\prime \prime} \lambda_{1} \cosh (x)+B^{\prime \prime} \lambda_{1} \sinh (x)+C^{\prime \prime}\right) .
$$


Rearranging (A.35b), (A.35c), and (A.35d) for the three regions (I, II, and III) shown in Figure 1, we have the three sets of expressions below

Region $\mathrm{I}(-l \leq x \leq-s)$

$$
\begin{gathered}
\sigma_{1 x}^{\imath}=A_{1} \sinh \left(\lambda_{1} x\right)+A_{2} \cosh \left(\lambda_{1} x\right)+A_{3} x+A_{4}, \\
\tau_{3 x}^{\imath}=-\delta_{0}\left(A_{1} \lambda_{1} \cosh \left(\lambda_{1} x\right)+A_{2} \lambda_{1} \sinh \left(\lambda_{1} x\right) \sinh +A_{3}\right), \\
\sigma_{3 y}^{\imath}=B_{1} \sinh \left(\omega_{1} x\right) \sin \left(\omega_{1} x\right)+B_{2} \sinh \left(\omega_{1} x\right) \cos \left(\omega_{1} x\right) \\
+B_{3} \cosh \left(\omega_{1} x\right) \sin \left(\omega_{1} x\right)+B_{4} \cosh \left(\omega_{1} x\right) \cos \left(\omega_{1} x\right) .
\end{gathered}
$$

Region II $(-s \leq x \leq s)$

$$
\begin{gathered}
\sigma_{1 x}^{u l}=A_{5} \sinh \left(\lambda_{2} x\right)+A_{6} \cosh \left(\lambda_{2} x\right)+A_{7} x+A_{8}, \\
\tau_{4 x}^{\imath l}=-\delta_{0}\left(A_{5} \lambda_{2} \cosh \left(\lambda_{2} x\right)+A_{6} \lambda_{2} \sinh \left(\lambda_{2} x\right)+A_{7}\right), \\
\sigma_{4 y}^{\imath l}=B_{5} \sinh \left(\omega_{2} x\right) \sin \left(\omega_{2} x\right)+B_{6} \sinh \left(\omega_{2} x\right) \cos \left(\omega_{2} x\right) \\
+B_{7} \cosh \left(\omega_{2} x\right) \sin \left(\omega_{2} x\right)+B_{8} \cosh \left(\omega_{2} x\right) \cos \left(\omega_{2} x\right) .
\end{gathered}
$$

Region III $(s \leq x \leq l)$

$$
\begin{gathered}
\sigma_{1 x}^{\imath u}=A_{9} \sinh \left(\lambda_{1} x\right)+A_{10} \cosh \left(\lambda_{1} x\right)+A_{11} x+A_{12}, \\
\tau_{3 x}^{\imath u l}=-\delta_{0}\left(A_{9} \lambda_{1} \cosh \left(\lambda_{1} x\right)+A_{10} \lambda_{1} \sinh \left(\lambda_{1} x\right)+A_{11}\right), \\
\sigma_{3 y}^{\imath \imath u}=B_{9} \sinh \left(\omega_{1} x\right) \sin \left(\omega_{1} x\right)+B_{10} \sinh \left(\omega_{1} x\right) \cos \left(\omega_{1} x\right) \\
+B_{11} \operatorname{csch}\left(\omega_{1} x\right) \sin \left(\omega_{1} x\right)+B_{12} \cosh \left(\omega_{1} x\right) \cos \left(\omega_{1} x\right)
\end{gathered}
$$

Set of (A.36a) and (A.36c) is related to the right and left flexible adhesive region. Set of (A.36b) is related to the stiff adhesive region, the central region. Normal, shear, and peel stresses are can be evaluated by using the appropriate expressions for each region.

Boundary Conditions (BCs). Continuity conditions of shear strain, normal strain, and slope at the interface between regions I and II lead to

$$
\begin{gathered}
\left.\frac{\tau_{3 x}^{\imath}}{G_{3}}\right|_{x=-s}=\left.\frac{\tau_{4 x}^{u}}{G_{4}}\right|_{x=-s}, \\
\left.\frac{\sigma_{3 y}^{\imath}}{E_{3}}\right|_{x=-s}=\left.\frac{\sigma_{4 y}^{u l}}{E_{4}}\right|_{x=-s}, \\
\left.\frac{\delta_{3}}{E_{3}} \frac{d \sigma_{3 y}^{\imath}}{d x}\right|_{x=-s}=\left.\frac{\delta_{3}}{E_{4}} \frac{d \sigma_{4 y}^{\imath l}}{d x}\right|_{x=-s} .
\end{gathered}
$$

The normal stress boundary conditions at the interfaces of the adhesives are

$$
\begin{gathered}
\left.\sigma_{1 x}^{u n}\right|_{x=s}=\left.\sigma_{1 x}^{u n}\right|_{x=s}, \\
\left.\sigma_{1 x}^{\imath}\right|_{x=-s}=\left.\sigma_{1 x}^{u n}\right|_{x=-s} .
\end{gathered}
$$

By using (A.17), (A.24), and (A.26), the boundary conditions at the interface between regions I and II may be written as

$$
\left.\left(\frac{\delta_{3}}{G_{3}}+\frac{2 \delta_{0}}{3 G}\right) \frac{d \tau_{3 x}^{\imath}}{d x}\right|_{x=-s}=\left.\left(\frac{\delta_{3}}{G_{4}}+\frac{2 \delta_{0}}{3 G}\right) \frac{d \tau_{4 x}^{\imath l}}{d x}\right|_{x=-s},
$$

$$
\begin{aligned}
& \left.\frac{\delta_{3}}{E_{3}} \frac{d^{2} \sigma_{3 y}^{\imath}}{d x^{2}}\right|_{x=-s}=\left.\frac{\delta_{3}}{E_{4}} \frac{d^{2} \sigma_{4 y}^{\imath l}}{d x^{2}}\right|_{x=-s}, \\
& \left.\frac{\delta_{3}}{E_{3}} \frac{d^{3} \sigma_{3 y}^{\imath}}{d x^{3}}\right|_{x=-s}=\left.\frac{\delta_{3}}{E_{4}} \frac{d^{3} \sigma_{4 y}^{\imath l}}{d x^{3}}\right|_{x=-s} .
\end{aligned}
$$

The boundary conditions at $x= \pm l$ for the moments, shear forces, and longitudinal forces are

$$
\begin{gathered}
\left\{\begin{array}{c}
\left.M_{1}\right|_{x=-l}=-M_{0} \\
\left.V_{1}\right|_{x=-l}=-V_{0} \\
\left.F_{1 x}\right|_{x=-l}=F
\end{array}\right\}, \quad\left\{\begin{array}{l}
\left.M_{2}\right|_{x=-l}=0 \\
\left.V_{2}\right|_{x=-l}=0 \\
\left.F_{2 x}\right|_{x=-l}=0
\end{array}\right\}, \\
\left\{\begin{array}{l}
\left.M_{1}\right|_{x=l}=0 \\
\left.V_{1}\right|_{x=l}=0 \\
\left.F_{1 x}\right|_{x=l}=0
\end{array}\right\}, \quad\left\{\begin{array}{l}
\left.M_{2}\right|_{x=l}=M_{0} \\
\left.V_{2}\right|_{x=l}=-V_{0} \\
\left.F_{1 x}\right|_{x=l}=F
\end{array}\right\} .
\end{gathered}
$$

Referring to Figure 1(b) and considering (A.39), the boundary conditions at $x=-l$ can be written by using (A.17), (A.18), (A.24), and (A.25) as

$$
\begin{gathered}
\left.\left(\frac{\delta_{3}}{G_{3}}+\frac{2 \delta_{0}}{3 G}\right) \frac{d \tau_{3 x}}{d x}\right|_{x=-l}=-\left(\frac{F}{E b \delta_{0}}+\frac{6 M_{0}}{E b \delta_{0}^{2}}\right) \\
\left.\left(\frac{\delta_{3}}{G_{3}}+\frac{2 \delta_{0}}{3 G}\right) \frac{d^{2} \tau_{3 x}}{d x^{2}}\right|_{x=-l}-\left.\frac{8}{E \delta_{0}} \tau_{3 x}\right|_{x=-l}=-\frac{6 V_{0}}{E b \delta_{0}^{2}}, \\
\left.\frac{\delta_{3}}{E_{3}} \frac{d^{2} \sigma_{3 y}}{d x^{2}}\right|_{x=-l}=\frac{12 M_{0}}{E b \delta_{0}^{3}} \\
\left.\frac{\delta_{3}}{E_{3}} \frac{d^{3} \sigma_{3 y}}{d x^{3}}\right|_{x=-l}=\frac{12 V_{0}}{E b \delta_{0}^{3}}
\end{gathered}
$$

The other two boundary conditions are

$$
\begin{aligned}
& \left.\sigma_{1 x}^{u n}\right|_{x=l}=\frac{\left.F_{1 x}\right|_{x=l}}{b \delta_{0}}=0, \\
& \left.\sigma_{1 x}^{\imath}\right|_{x=-l}=\frac{\left.F_{1 x}\right|_{x=-l}}{b \delta_{1}}=\frac{F}{b \delta_{0}} .
\end{aligned}
$$


In (A.36a), (A.36b), and (A.36c) there are a total of 24 unknown coefficients corresponding to the three regions. However, we constructed only 14 BCs above. Ten boundary conditions are needed for the problem at hand. We wrote the boundary conditions above for the left group of flexible-stiff adhesive layer (regions I and II). From $y$-axis symmetry, we can again write additional new boundary conditions for the right group of stiff-flexible adhesive layer (regions II and III). Then, the 24 unknown coefficients can be obtained from these BCs.

\section{Conflict of Interests}

The authors declare that there is no conflict of interests regarding the publication of this paper.

\section{Acknowledgment}

The authors are grateful to reviewers for their valuable comments on this paper.

\section{References}

[1] R. D. Adams and N. A. Peppiatt, "Effect of poisson's ratio strains in adherends on stresses of an idealized lap joint," The Journal of Strain Analysis for Engineering Design, vol. 8, no. 2, pp. 134-139, 1973.

[2] L. J. Hart-Smith, "Adhesive-bonded single-lap joints," NASA Report CR-112236, NASA, Washington, DC, USA, 1973.

[3] R. D. Adams and N. A. Peppiatt, "Stress analysis of adhesivebonded lap joints," The Journal of Strain Analysis for Engineering Design, vol. 9, no. 3, pp. 185-196, 1974.

[4] D. J. Allman, "A theory for elastic stresses in adhesive bonded lap joints," The Quarterly Journal of Mechanics \& Applied Mathematics, vol. 30, no. 4, pp. 415-436, 1977.

[5] D. A. Bigwood and A. D. Crocombe, "Elastic analysis and engineering design formulae for bonded joints," International Journal of Adhesion \& Adhesives, vol. 9, no. 4, pp. 229-242, 1989.

[6] M. Y. Tsai, D. W. Oplinger, and J. Morton, "Improved theoretical solutions for adhesive lap joints," International Journal of Solids and Structures, vol. 35, no. 12, pp. 1163-1185, 1998.

[7] B. Zhao and Z.-H. Lu, "A two-dimensional approach of singlelap adhesive bonded joints," Mechanics of Advanced Materials and Structures, vol. 16, no. 2, pp. 130-159, 2009.

[8] B. Zhao, Z.-H. Lu, and Y.-N. Lu, "Closed-form solutions for elastic stressstrain analysis in unbalanced adhesive single-lap joints considering adherend deformations and bond thickness," International Journal of Adhesion \& Adhesives, vol. 31, no. 6, pp. 434-445, 2011.

[9] C. Raphael, "Variable-adhesive bonded joints," Applied Polymer Symposia, vol. 3, pp. 99-108, 1966.

[10] S. Srinivas, "Analysis of bonded joints," NASA Report TN D7855, NASA, Washington, DC, USA, 1975.

[11] I. Pires, L. Quintino, J. F. Durodola, and A. Beevers, "Performance of bi-adhesive bonded aluminium lap joints," International Journal of Adhesion \& Adhesives, vol. 23, no. 3, pp. 215223, 2003.

[12] M. D. Fitton and J. G. Broughton, "Variable modulus adhesives: an approach to optimised joint performance," International
Journal of Adhesion \& Adhesives, vol. 25, no. 4, pp. 329-336, 2005.

[13] F.-R. Kong, M. You, and X.-L. Zheng, “Three-dimensional finite element analysis of the stress distribution in bi-adhesive bonded joints," The Journal of Adhesion, vol. 84, no. 2, pp. 105-124, 2008.

[14] S. Kumar, "Analysis of tubular adhesive joints with a functionally modulus graded bondline subjected to axial loads," International Journal of Adhesion \& Adhesives, vol. 29, no. 8, pp. 785-795, 2009.

[15] L. F. M. da Silva and M. C. Q. Lopes, "Joint strength optimization by the mixed-adhesive technique," International Journal of Adhesion \& Adhesives, vol. 29, no. 5, pp. 509-514, 2009.

[16] M. You, J. Yan, X. Zheng, D. Zhu, J. Li, and J. Hu, "The numerical analysis on the weld-bonded joints with bi-adhesive," in Proceedings of the 2nd International Conference on Computer Modeling and Simulation (ICCMS '10), pp. 25-28, Sanya, China, January 2010.

[17] L. F. M. da Silva and R. D. Adams, "Adhesive joints at high and low temperatures using similar and dissimilar adherends and dual adhesives," International Journal of Adhesion \& Adhesives, vol. 27, no. 3, pp. 216-226, 2007.

[18] S. Temiz, "Application of bi-adhesive in double-strap joints subjected to bending moment," Journal of Adhesion Science and Technology, vol. 20, no. 14, pp. 1547-1560, 2006.

[19] P. J. C. das Neves, L. F. M. da Silva, and R. D. Adams, "Analysis of mixed adhesive bonded joints part I: theoretical formulation," Journal of Adhesion Science and Technology, vol. 23, no. 1, pp. 1-34, 2009.

[20] I. Pires, L. Quintino, and R. M. Miranda, "Numerical simulation of mono- and bi-adhesive aluminium lap joints," Journal of Adhesion Science and Technology, vol. 20, no. 1, pp. 19-36, 2006.

[21] S. Kumar and P. C. Pandey, "Behaviour of Bi-adhesive joints," Journal of Adhesion Science and Technology, vol. 24, no. 7, pp. 1251-1281, 2010.

[22] R. J. C. Carbas, L. F. M. da Silva, and G. W. Critchlow, "Adhesively bonded functionally graded joints by induction heating," International Journal of Adhesion \& Adhesives, vol. 48, pp. 110-118, 2014.

[23] R. J. C. Carbas, L. F. M. da Silva, M. L. Madureira, and G. W. Critchlow, "Modelling of functionally graded adhesive joints," The Journal of Adhesion, 2013.

[24] O. Bavi, N. Bavi, and M. Shishesaz, "Geometrical optimization of the overlap in mixed adhesive lap joints," The Journal of Adhesion, vol. 89, no. 12, pp. 948-972, 2013.

[25] O. Volkersen, "Die niektraftverteilung in zugbeanspruchten mit konstanten laschenquerschritten," Luftfahrtforschung, vol. 15, pp. 41-47, 1938.

[26] L. F. M. da Silva, P. J. C. das Neves, R. D. Adams, and J. K. Spelt, "Analytical models of adhesively bonded joints-part I: literature survey," International Journal of Adhesion \& Adhesives, vol. 29, no. 3, pp. 319-330, 2009.

[27] L. F. M. da Silva, P. J. C. das Neves, R. D. Adams, A. Wang, and J. K. Spelt, "Analytical models of adhesively bonded jointspart II: comparative study," International Journal of Adhesion \& Adhesives, vol. 29, no. 3, pp. 331-341, 2009.

[28] B. Zhao, "Theoretical stress model of mixed-modulus single-lap adhesive bonded joints considering the bending effect," Chinese Journal of Mechanical Engineering, vol. 44, no. 10, pp. 129-137, 2008.

[29] D. A. Dillard and A. V. Pocius, Adhesion Science and Engineering-I: The Mechanics of Adhesion, Elsevier Science, Amsterdam, The Netherlands, 2002. 
[30] J. P. M. Gonçalves, M. F. S. F. de Moura, and P. M. S. T. de Castro, "A three-dimensional finite element model for stress analysis of adhesive joints," International Journal of Adhesion \& Adhesives, vol. 22, no. 5, pp. 357-365, 2002.

[31] H. Özer and Ö. Öz, "Three dimensional finite element analysis of bi-adhesively bonded double lap joint," International Journal of Adhesion \& Adhesives, vol. 37, pp. 50-55, 2012.

[32] G. P. Zou, K. Shahin, and F. Taheri, "An analytical solution for the analysis of symmetric composite adhesively bonded joints," Composite Structures, vol. 65, no. 3-4, pp. 499-510, 2004. 


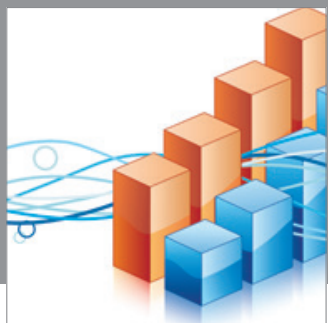

Advances in

Operations Research

mansans

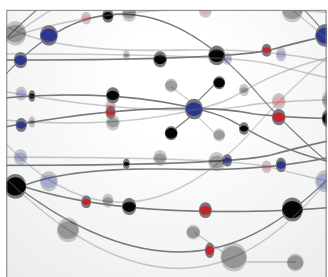

The Scientific World Journal
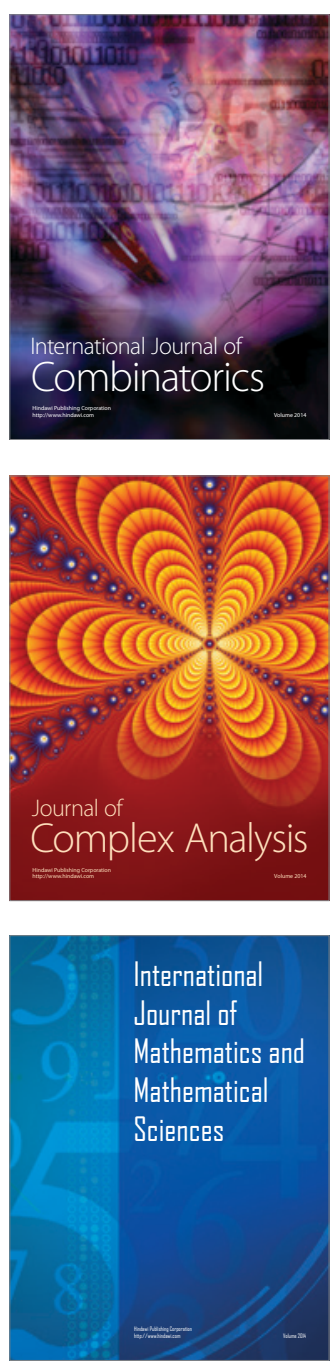
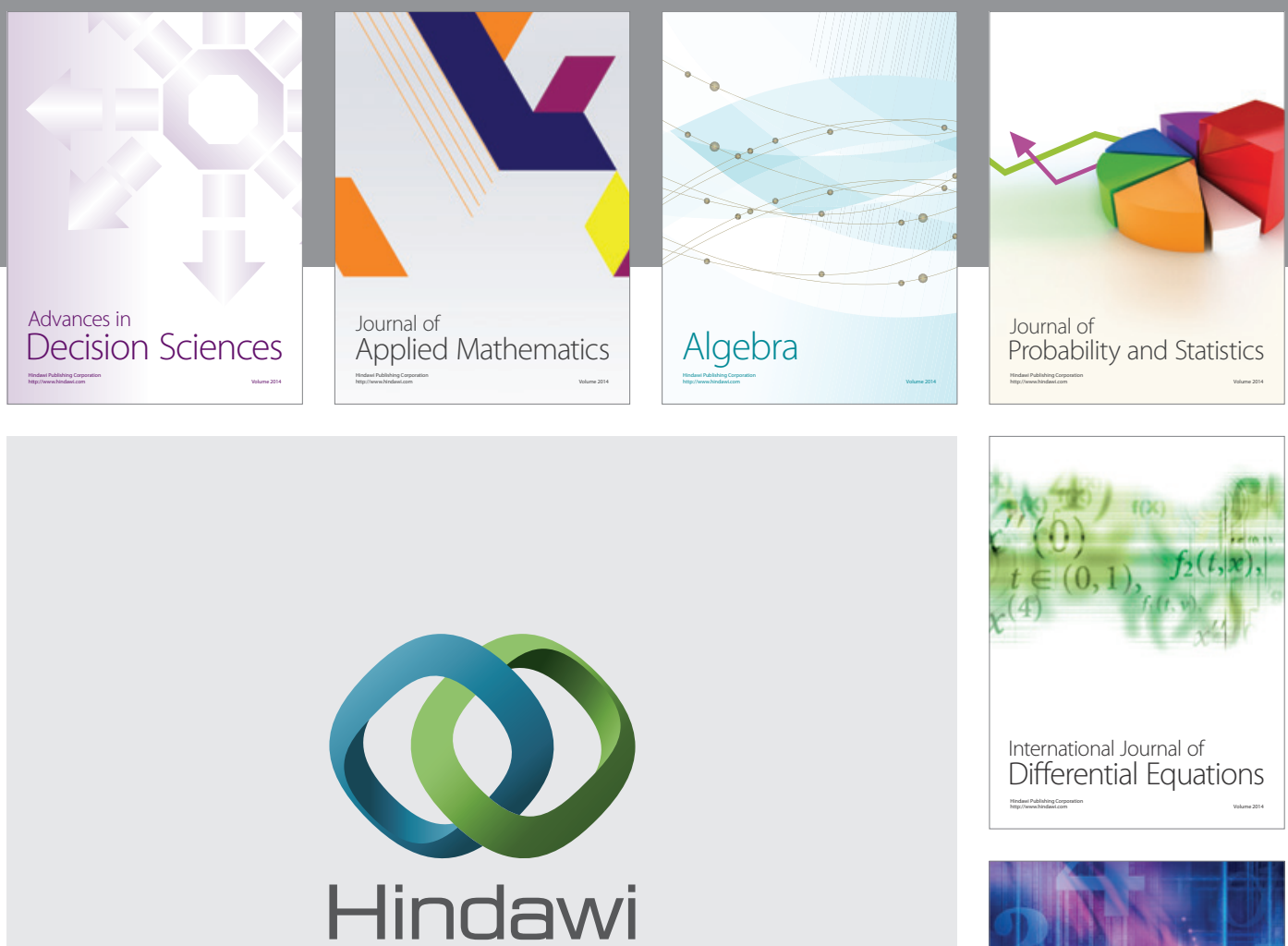

Submit your manuscripts at http://www.hindawi.com
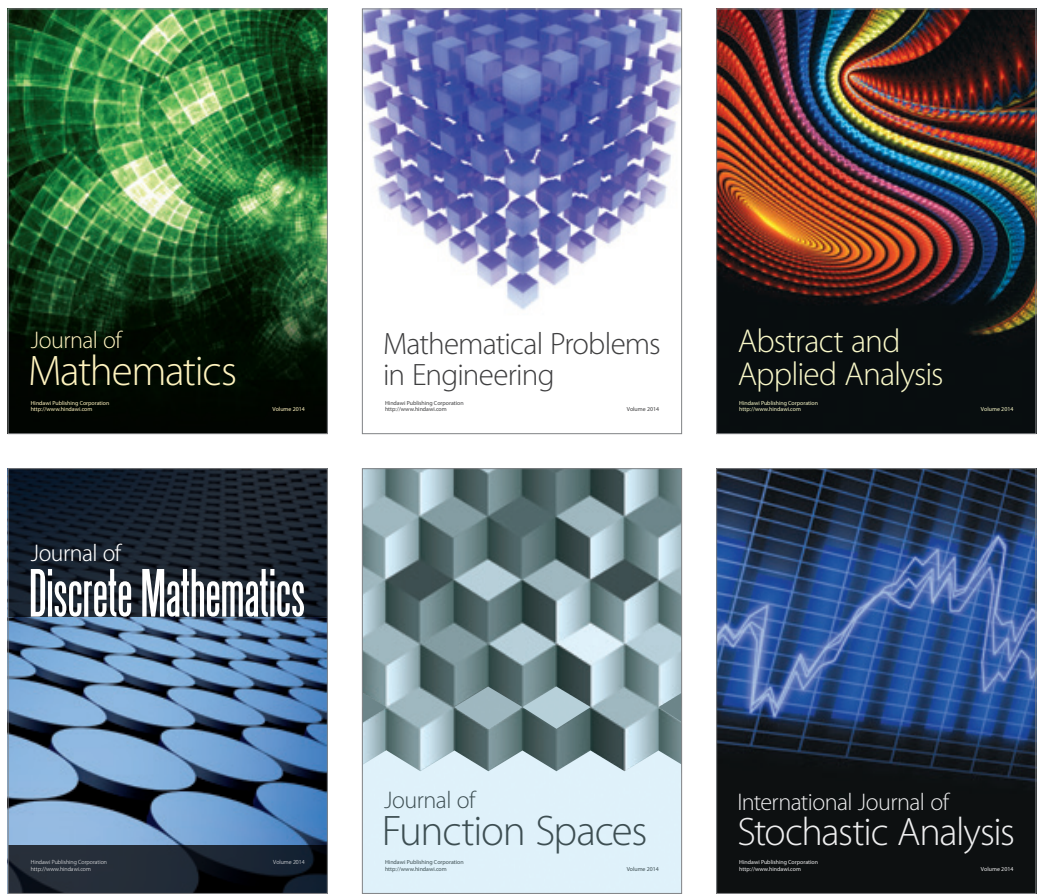

Journal of

Function Spaces

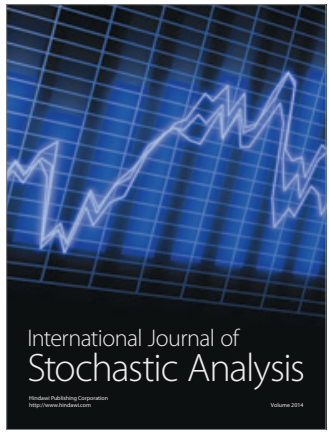

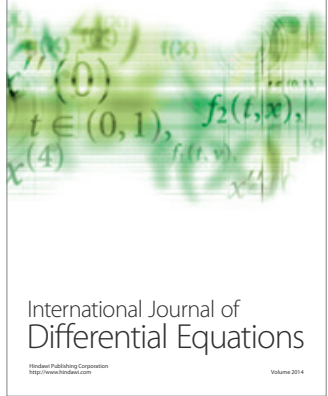
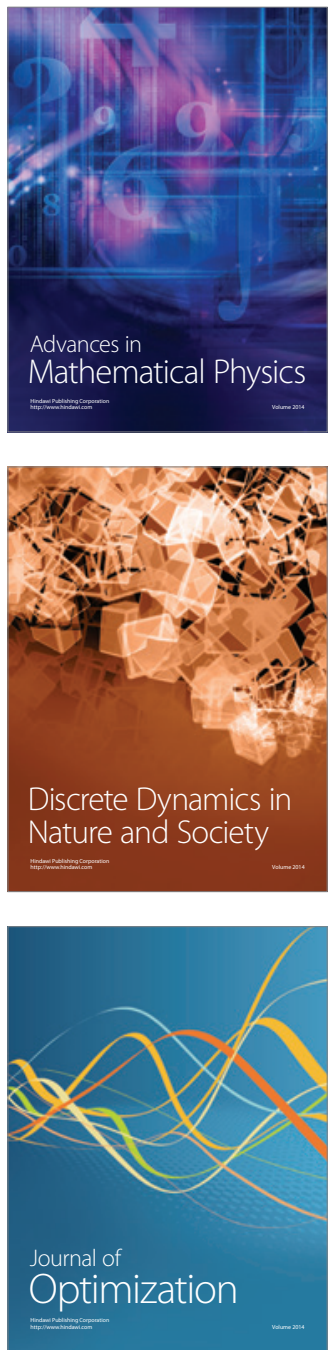